\title{
Isodiametric sets in the Heisenberg group
}

\author{
Gian Paolo Leonardi, Séverine Rigot and Davide Vittone
}

\begin{abstract}
In the sub-Riemannian Heisenberg group equipped with its Carnot-Carathéodory metric and with a Haar measure, we consider isodiametric sets, i.e., sets maximizing measure among all sets with a given diameter. In particular, given an isodiametric set, and up to negligible sets, we prove that its boundary is given by the graphs of two locally Lipschitz functions. Moreover, within the restricted class of rotationally invariant sets, we give a quite complete characterization of any compact (rotationally invariant) isodiametric set. More precisely, its Steiner symmetrization with respect to the $\mathbb{C}^{n}$-plane is shown to coincide with the Euclidean convex hull of a CC-ball. At the same time, we also prove quite unexpected non-uniqueness results.
\end{abstract}

\section{Introduction}

The classical isodiametric inequality in the Euclidean space says that balls have maximal volume among all sets with a given diameter. This was originally proved by Bieberbach [5] in $\mathbb{R}^{2}$ and by Urysohn [14] in $\mathbb{R}^{n}$, see also [6]. In this paper we are interested in the case of the Heisenberg group $\mathbb{H}^{n}$ equipped with its CarnotCarathéodory distance $d$ and with the Haar measure $\mathcal{L}^{2 n+1}$ (see Section 2 for the definitions). Our aim is to study isodiametric sets, i.e., sets maximizing the measure among sets with a given diameter.

Recalling that the homogeneous dimension of $\mathbb{H}^{n}$ is $2 n+2$, we define the isodiametric constant $C_{I}$ by

$$
C_{I}=\sup \mathcal{L}^{2 n+1}(F) /(\operatorname{diam} F)^{2 n+2}
$$

where the supremum is taken over all sets $F \subset \mathbb{H}^{n}$ with positive and finite diameter. Sets realizing the supremum do exist, see [12] or Theorem 3.1 below. Since the closure of any such set is a compact set that still realizes the supremum, we consider the class $\mathcal{I}$ of compact isodiametric sets,

$$
\mathcal{I}=\left\{E \subset \mathbb{H}^{n}: E \text { compact, } \operatorname{diam} E>0, \mathcal{L}^{2 n+1}(E)=C_{I}(\operatorname{diam} E)^{2 n+2}\right\} .
$$

Mathematics Subject Classification (2010): 53C17, 28A75, 49Q15, 22E30.

Keywords: Isodiametric problem, Heisenberg group. 
In other words, $\mathcal{I}$ denotes the class of compact sets that maximize $\mathcal{L}^{2 n+1}$-measure among all sets with the same diameter.

In contrast to the Euclidean case, balls in $\left(\mathbb{H}^{n}, d\right)$ are not isodiametric (see [12]) and we shall give in this paper some further and refined evidence that the situation is indeed quite different from the Euclidean one.

Before describing our main results let us recall some classical motivations and consequences coming from the study of isodiametric type problems. First the isodiametric constant $C_{I}$ coincides with the ratio between the measure $\mathcal{L}^{2 n+1}$ and the $(2 n+2)$-dimensional Hausdorff measure $\mathcal{H}^{2 n+2}$ in $\left(\mathbb{H}^{n}, d\right)$, namely,

$$
\mathcal{L}^{2 n+1}=C_{I} \mathcal{H}^{2 n+2},
$$

where $\mathcal{H}^{2 n+2}(A)=\lim _{\delta \downarrow 0} \inf \left\{\sum_{i}\left(\operatorname{diam} A_{i}\right)^{2 n+2}: A \subset \cup_{i} A_{i}, \operatorname{diam} A_{i} \leq \delta\right\}$. This can actually be generalized to any Carnot group equipped with a homogeneous distance (see [12]), and for abelian Carnot groups one recovers the well-known Euclidean situation. We also refer the interested reader to [1] where some relationships between different intrinsic volumes that can be defined in sub-Riemannian geometry are studied.

As a consequence, the knowledge of the numerical value of the isodiametric constant $C_{I}$, or equivalently the explicit description of isodiametric sets, gives non-trivial information about the geometry of the metric space $\left(\mathbb{H}^{n}, d\right)$ and about the measure $\mathcal{H}^{2 n+2}$ which may be considered as a natural measure from the metric point of view.

There are also some links with the Besicovitch 1/2-problem which is in turn related to the study of the connections between densities and rectifiability. Let us sketch this connection briefly here. We refer to [11] for a more detailed introduction and known results about the Besicovitch 1/2-problem and [12] for more details about the connection between the isodiametric problem in Carnot groups and the Besicovitch 1/2-problem. Let $\sigma_{n}(M, d)$ denote the density constant of the metric space $(M, d)$. It is the smallest number such that every subset with finite $\mathcal{H}^{n}$-measure having $n$-dimensional lower density strictly greater than $\sigma_{n}(M, d)$ at $\mathcal{H}^{n}$-almost all of its points is $n$-rectifiable (see [11] for the precise definition). The validity of the bound $\sigma_{n}(M, d) \leq 1 / 2$ for any separable metric space $(M, d)$, which was conjectured long ago by A.S. Besicovitch for the one-dimensional density constant in $\mathbb{R}^{2}$ (see [4]), is known as the generalized Besicovitch $1 / 2$-problem. It turns out that for a Carnot group equipped with a homogeneous distance $(G, d)$ and with homogeneous dimension $Q$, the density constant $\sigma_{Q}(G, d)$ can be easily related to the inverse of the isodiametric constant. Then upper bounds on the isodiametric constant give lower bounds on $\sigma_{Q}(G, d)$. Following these ideas it is in particular proved in $[12]$ that $\sigma_{2 n+2}\left(\mathbb{H}^{n}, d\right)>1 / 2$ for $n \in\{1, \ldots, 8\}$, thus giving non-trivial counterexamples to the Besicovitch $1 / 2$-conjecture. It is clear that the precise knowledge of the numerical value of the isodiametric constant would help in obtaining further and more complete results about the Besicovitch 1/2-problem.

Our main results in the present paper are a regularity property for sets in $\mathcal{I}$ and a rather complete solution to a restricted isodiametric problem within the class of so-called rotationally invariant sets. 
Let us first describe our regularity result. We shall prove that, given $E \in \mathcal{I}$, then $\overline{\text { int } E}$ is still a compact isodiametric set with the same diameter as $E$ and with locally Lipschitz boundary. More precisely, identifying $\mathbb{H}^{n}$ with $\mathbb{C}^{n} \times \mathbb{R}$ (see Section 2), we prove that

$$
\overline{\operatorname{int} E}=\left\{[z, t] \in \mathbb{H}^{n}: z \in \bar{U}, f^{-}(z) \leq t \leq f^{+}(z)\right\}
$$

for some open set $U$ in $\mathbb{C}^{n}$ and some continuous maps $f^{-}, f^{+}: \bar{U} \rightarrow \mathbb{R}$ that are locally Lipschitz continuous on $U$. See Theorem 3.12 for a complete statement.

This regularity property will actually follow from a slightly more general result. We will prove that a set $E \in \mathcal{I}$ must satisfy the following necessary condition:

$$
\text { for all } p \in \partial E \text {, there exists } q \in \partial E \text { such that } d(p, q)=\operatorname{diam} E \text {, }
$$

see Proposition 3.2, and is $t$-convex, see Subsection 2.4 for the definition of $t$-convexity and Proposition 3.11. Independently of the isodiametric problem, the property (NC) together with $t$-convexity turn out to imply the regularity properties sketched above. See Theorem 3.3.

As already mentioned, one knows that balls in $\left(\mathbb{H}^{n}, d\right)$ are not isodiametric, and isodiametric sets in $\left(\mathbb{H}^{n}, d\right)$ are actually not explicitly known. This question turns out to be a challenging and rather delicate one. However, restricting ourselves to the family $\mathcal{R}$ of so-called rotationally invariant sets, we are able to give a rather complete picture of the situation for compact isodiametric sets within this class. As we shall explain below, this picture will give some further information about the class $\mathcal{I}$. This may also hopefully give some insight towards a complete solution of the general isodiametric problem in $\left(\mathbb{H}^{n}, d\right)$.

We shall denote by $\mathcal{I}_{\mathcal{R}}$ the class of compact sets in $\mathcal{R}$ that are isodiametric within the class $\mathcal{R}$. See Section 3 for the definition of the class $\mathcal{R}$ and of this restricted isodiametric problem. First it is not hard to check that sets in $\mathcal{I}_{\mathcal{R}}$ satisfy (NC) and are $t$-convex, see Propositions 3.2 and 3.11, and hence satisfy the regularity properties of Theorem 3.3. Next our main specific result concerning sets $E \in \mathcal{I}_{\mathcal{R}}$ is the characterization of their Steiner symmetrization St $E$ with respect to the $\mathbb{C}^{n}$-plane (see Subsection 2.4 for the definition of St $E$ ). We prove that if $E \in \mathcal{I}_{\mathcal{R}}$ then $\mathrm{St} E$ belongs to $\mathcal{I}_{\mathcal{R}}$ and has the same diameter as $E$. Moreover we prove that St $E$ is actually uniquely determined once the diameter of $E$ is fixed, i.e., St $E=A_{\text {diam } E}$ for some particular set $A_{\text {diam } E}$, see Theorem 4.4.

Given $\lambda>0$, the set $A_{\lambda}$ can be guessed via the following argument. One starts with the ball in $\left(\mathbb{H}^{n}, d\right)$ centered at the origin and with diameter $\lambda$. As already said it does not satisfy (NC). Thus one can enlarge it around points where (NC) fails and get a set with still the same diameter but with greater measure. One can actually try to enlarge it as much as possible without increasing the diameter, remaining in the class $\mathcal{R}$, and preserving the property that it coincides with its Steiner symmetrization with respect to the $\mathbb{C}^{n}$-plane. In such a way, one ends up with a maximal set $A_{\lambda}$ that satisfies (NC). It turns out that this set is the closed convex hull (in the Euclidean sense when identifying $\mathbb{H}^{n}$ with $\mathbb{R}^{2 n+1}$ ) of the ball in $\left(\mathbb{H}^{n}, d\right)$ centered at the origin and with diameter $\lambda$. See Figure 1. 

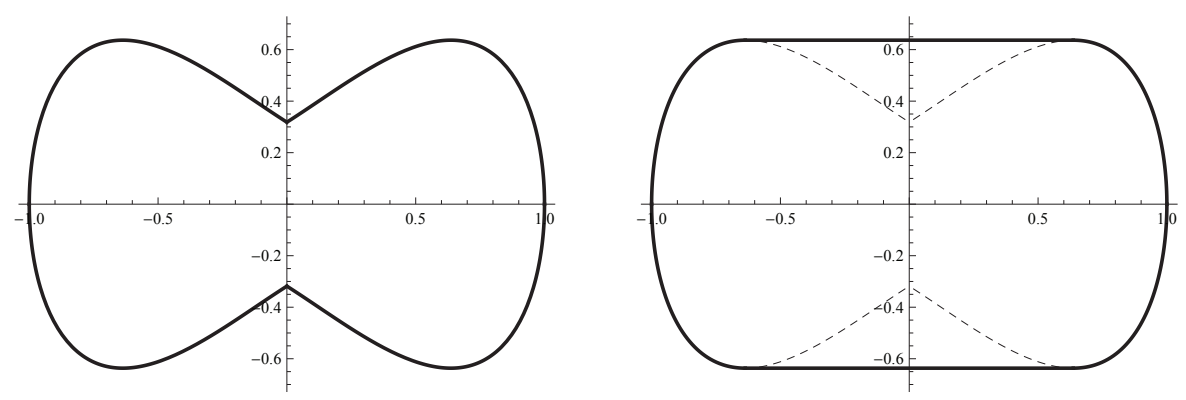

FIGURE 1. The intersection of the boundary $\partial B(0,1)$ of the unit ball (left) and of the boundary $\partial_{2}$ of its Euclidean convex hull (right) with a plane containing the vertical $t$-axis.

We also construct small suitable perturbations of the set $A_{\lambda}$ that preserve its Lebesgue measure and its diameter, see Proposition 4.5. Considering rotationally invariant perturbations, this gives the non uniqueness of sets in $\mathcal{I}_{\mathcal{R}}$. This non uniqueness has to be understood in an "essential" sense, i.e., also up to left translations and dilations. See Corollary 4.6.

Finally, considering not rotationally invariant perturbations, one gets the existence of sets in $\mathcal{I}$ that are not rotationally invariant, even modulo left translations, see Corollary 4.7. This gives one more significant difference with the Euclidean situation. See Figure 2.

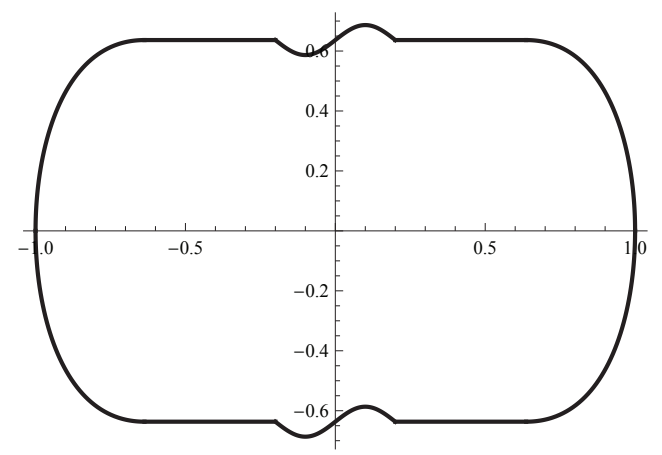

FIgURE 2. The intersection of the boundary of a perturbation of $A_{2}$ with a plane containing the vertical $t$-axis.

The paper is organized as follows. In Section 2 we introduce the Heisenberg group $\mathbb{H}^{n}$ and recall basic facts about the Carnot-Carathéodory distance $d$ and balls in $\left(\mathbb{H}^{n}, d\right)$. We also introduce $t$-convexification and the Steiner symmetrization with respect to the $\mathbb{C}^{n}$-plane. Section 3 is devoted to existence and regularity results, while in Section 4 we prove our more specific results about the isodiametric problem restricted to the class $\mathcal{R}$. 


\section{Notation and preliminary results}

\subsection{The Heisenberg group}

The Heisenberg group $\mathbb{H}^{n}$ is a connected and simply connected Lie group with stratified Lie algebra (see, e.g., [13], [7]). We identify it with $\mathbb{C}^{n} \times \mathbb{R}$ and denote points in $\mathbb{H}^{n}$ by $[z, t]$, where $z=\left(z_{1}, \ldots, z_{n}\right) \in \mathbb{C}^{n}$ and $t \in \mathbb{R}$. The group law is

$$
[z, t] \cdot\left[z^{\prime}, t^{\prime}\right]=\left[z+z^{\prime}, t+t^{\prime}+2 \operatorname{Im} z \bar{z}^{\prime}\right],
$$

where $\operatorname{Im} z \bar{z}^{\prime}=\sum_{j=1}^{n} \operatorname{Im} z_{j} \bar{z}_{j}^{\prime}$. The unit element is $0=[0,0]$.

There is a natural family of dilations $\delta_{\lambda}$ on $\mathbb{H}^{n}$ defined by $\delta_{\lambda}([z, t])=\left[\lambda z, \lambda^{2} t\right]$ for $\lambda \geq 0$.

We define the canonical projection $\pi: \mathbb{H}^{n} \rightarrow \mathbb{C}^{n}$ as

$$
\pi([z, t])=z
$$

for any $[z, t] \in \mathbb{H}^{n}$. Given $p \in \mathbb{H}^{n}$, we shall frequently denote by $\left[z_{p}, t_{p}\right]$ its coordinates in $\mathbb{C}^{n} \times \mathbb{R}$.

We also represent $\mathbb{H}^{n}$ as $\mathbb{R}^{2 n+1} \approx \mathbb{R}^{n} \times \mathbb{R}^{n} \times \mathbb{R}$ through the identification $[z, t] \approx(x, y, t)$, where $x=\left(x_{1}, \ldots, x_{n}\right), y=\left(y_{1}, \ldots, y_{n}\right) \in \mathbb{R}^{n}, t \in \mathbb{R}$ and $z=$ $\left(z_{1}, \ldots, z_{n}\right) \in \mathbb{C}^{n}$ with $z_{j}=x_{j}+i y_{j}$

The $(2 n+1)$-dimensional Lebesgue measure $\mathcal{L}^{2 n+1}$ on $\mathbb{H}^{n} \approx \mathbb{R}^{2 n+1}$ is a Haar measure of the group. It is $(2 n+2)$-homogeneous with respect to dilations,

$$
\mathcal{L}^{2 n+1}\left(\delta_{\lambda}(F)\right)=\lambda^{2 n+2} \mathcal{L}^{2 n+1}(F)
$$

for all measurable $F \subset \mathbb{H}^{n}$ and $\lambda \geq 0$.

The horizontal subbundle of the tangent bundle is defined by

$$
\mathcal{H}^{n}=\operatorname{span}\left\{X_{j}, Y_{j}: j=1, \ldots, n\right\}
$$

where the left invariant vectors fields $X_{j}$ and $Y_{j}$ are given by

$$
X_{j}=\partial_{x_{j}}+2 y_{j} \partial_{t}, \quad Y_{j}=\partial_{y_{j}}-2 x_{j} \partial_{t} .
$$

Setting $T=\partial_{t}$ the only non trivial bracket relations are $\left[X_{j}, Y_{j}\right]=-4 T$, hence the Lie algebra of $\mathbb{H}^{n}$ admits the stratification $\mathcal{H}^{n} \oplus \operatorname{span}\{T\}$.

\subsection{Carnot-Carathéodory distance}

We fix a left invariant Riemannian metric $g$ on $\mathbb{H}^{n}$ with $\left(X_{1}, \ldots, X_{n}, Y_{1}, \ldots, Y_{n}, T\right)$ as an orthonormal basis. The Carnot-Carathéodory distance between any two points $p$ and $q \in \mathbb{H}^{n}$ is then defined by

$$
d(p, q)=\inf \left\{\operatorname{length}_{g}(\gamma): \gamma \text { horizontal curve joining } p \text { to } q\right\},
$$

where a curve is said to be horizontal if it is absolutely continuous and such that at a.e. every point its tangent vector belongs to the horizontal subbundle $\mathcal{H}^{n}$ of 
the tangent bundle. Recall that by the Chow-Rashevsky theorem, any two points can be joined by a horizontal curve of finite length. Therefore the function $d$ turns out to be a distance. It induces the original topology of the group, it is left-invariant, i.e.,

$$
d\left(p \cdot q, p \cdot q^{\prime}\right)=d\left(q, q^{\prime}\right)
$$

for all $p, q, q^{\prime} \in \mathbb{H}^{n}$, and has homogeneity one with respect to dilations, i.e.,

$$
d\left(\delta_{\lambda}(p), \delta_{\lambda}(q)\right)=\lambda d(p, q)
$$

for all $p, q \in \mathbb{H}^{n}$ and $\lambda \geq 0$.

Equipped with this distance, $\mathbb{H}^{n}$ is a separable and complete metric space in which closed bounded sets are compact. We will denote by $B(p, r)$, respectively $\bar{B}(p, r)$, the open, respectively closed, ball with center $p \in \mathbb{H}^{n}$ and radius $r>0$. Note that the diameter of any ball in $\left(\mathbb{H}^{n}, d\right)$ is given by twice its radius.

Lemma 2.1. Let $p \in \mathbb{H}^{n}$. The distance function $d_{p}: \mathbb{H}^{n} \backslash\{p\} \rightarrow \mathbb{R}$ from $p$ defined by $d_{p}(q)=d(p, q)$ is an open map.

Proof. We prove that $d_{p}(B)$ is open for any open ball $B \subset \mathbb{H}^{n} \backslash\{p\}$. Since balls in $\left(\mathbb{H}^{n}, d\right)$ are connected (this is more generally true in any length space) it follows that $d_{p}(B)$ is a bounded interval. Setting $m=\inf \left(d_{p}(q): q \in B\right)$ and $M=$ $\sup \left(d_{p}(q): q \in B\right)$, it is thus enough to prove that $m \notin d_{p}(B)$ and $M \notin d_{p}(B)$.

If $m=0$ then $m \notin d_{p}(B)$ because $p \notin B$. If $m>0$ we assume by contradiction that $m \in d_{p}(B)$. Then we can find $q \in B$ such that $d_{p}(q)=m$. The map $\lambda \in[0,+\infty) \mapsto p \cdot \delta_{\lambda}\left(p^{-1} \cdot q\right)$ being continuous, one has $p \cdot \delta_{\lambda}\left(p^{-1} \cdot q\right) \in B$ for all $\lambda$ close enough to 1 . It follows that

$$
d_{p}(q) \leq d_{p}\left(p \cdot \delta_{\lambda}\left(p^{-1} \cdot q\right)\right)=\lambda d_{p}(q)<d_{p}(q)
$$

provided $\lambda<1$ is close enough to 1 , which gives a contradiction. The fact that $M \notin d_{p}(B)$ can be proved in a similar way and this concludes the proof.

Remark 2.2. As an immediate consequence of Lemma 2.1 we get that for any set $F \subset \mathbb{H}^{n}$ and any $p \in F$,

$$
d(p, q)<\operatorname{diam} F \quad \text { for all } q \in \operatorname{int} F .
$$

Moreover, if $F$ is bounded then $\operatorname{diam} F=\operatorname{diam} \partial F$.

Although the Carnot-Carathéodory distance between any two points is in general hardly explicitly computable, we recall for further reference the following well known special cases. One has

$$
d\left([z, t],\left[z^{\prime}, t\right]\right)=\left\|z^{\prime}-z\right\|
$$

for all $z, z^{\prime} \in \mathbb{C}^{n}$ such that $\operatorname{Im} z \bar{z}^{\prime}=0$ and all $t \in \mathbb{R}$. Here $\|z\|=\left(\sum_{j=1}^{n}\left|z_{j}\right|^{2}\right)^{1 / 2}$ for $z=\left(z_{1}, \ldots, z_{n}\right) \in \mathbb{C}^{n}$. We have also

$$
d\left([z, t],\left[z, t^{\prime}\right]\right)=\left(\pi\left|t^{\prime}-t\right|\right)^{1 / 2}
$$

for all $z \in \mathbb{C}^{n}$ and $t, t^{\prime} \in \mathbb{R}$. 


\subsection{Description of balls and consequences}

Explicit descriptions of balls in $\left(\mathbb{H}^{n}, d\right)$ are well known, see [2], [9], [10]. One has

$$
\bar{B}(0,1)=\left\{\left[\frac{\sin \varphi}{\varphi} \chi, \frac{2 \varphi-\sin (2 \varphi)}{2 \varphi^{2}}\|\chi\|^{2}\right] \in \mathbb{H}^{n}: \chi \in \mathbb{C}^{n},\|\chi\| \leq 1, \varphi \in[-\pi, \pi]\right\} .
$$

We set

$$
g(\varphi)= \begin{cases}\frac{2 \varphi-\sin (2 \varphi)}{2 \varphi^{2}} & \text { for } 0<\varphi \leq \pi \\ 0 & \text { if } \varphi=0\end{cases}
$$

The function $g$ has a maximum at $\varphi=\pi / 2$ with $g(\pi / 2)=2 / \pi$. It is increasing from $[0, \pi / 2]$ onto $[0,2 / \pi]$ and decreasing from $[\pi / 2, \pi]$ onto $[1 / \pi, 2 / \pi]$.

We set

$$
\rho(\varphi)= \begin{cases}\frac{\sin \varphi}{\varphi} & \text { for } 0<\varphi \leq \pi \\ 1 & \text { if } \varphi=0 .\end{cases}
$$

The function $\rho$ is decreasing from $[0, \pi]$ onto $[0,1]$. Let $\rho^{-1}:[0,1] \rightarrow[0, \pi]$ denote its inverse and set

$$
h=g \circ \rho^{-1} .
$$

We have the following alternative description of the closed unit ball:

$$
\bar{B}(0,1)=\left\{[z, t] \in \mathbb{H}^{n}:\|z\| \leq 1,|t| \leq h(\|z\|)\right\} .
$$

Using dilations we have

$$
\bar{B}(0, \lambda)=\left\{[z, t] \in \mathbb{H}^{n}:\|z\| \leq \lambda,|t| \leq h_{\lambda}(\|z\|)\right\}
$$

for all $\lambda>0$, where

$$
h_{\lambda}(\|z\|)=\lambda^{2} h(\|z\| / \lambda) .
$$

We list some properties of the function $h$ that will be needed in the sequel. See Figure 3 for a picture.

(i) The map $h$ is increasing from $[0,2 / \pi]$ onto $[1 / \pi, 2 / \pi]$ and decreasing from $[2 / \pi, 1]$ onto $[0,2 / \pi]$.

(ii) There exists $r_{c} \in(0,2 / \pi)$ such that $h^{\prime \prime}>0$ on $\left(0, r_{c}\right), h^{\prime \prime}\left(r_{c}\right)=0$, and $h^{\prime \prime}<0$ on $\left(r_{c}, 1\right)$.

Indeed we have

$$
h^{\prime}(r)=-2 \frac{\cos \varphi}{\varphi} \quad \text { and } \quad h^{\prime \prime}(r)=2 \frac{\varphi \sin \varphi+\cos \varphi}{\varphi^{2} \rho^{\prime}(\varphi)}
$$

for all $r \in[0,1)$ and where $\varphi=\rho^{-1}(r) \in(0, \pi]$. Then statement (i) follows from the expression of $h^{\prime}$ together with the properties of $\rho^{-1}$. Statement (ii) follows noting that there exists a unique $\varphi_{c} \in(\pi / 2, \pi)$ such that $\varphi_{c} \sin \varphi_{c}+\cos \varphi_{c}=0$ and that one has $\varphi \sin \varphi+\cos \varphi>0$ if and only if $0<\varphi<\varphi_{c}$. 


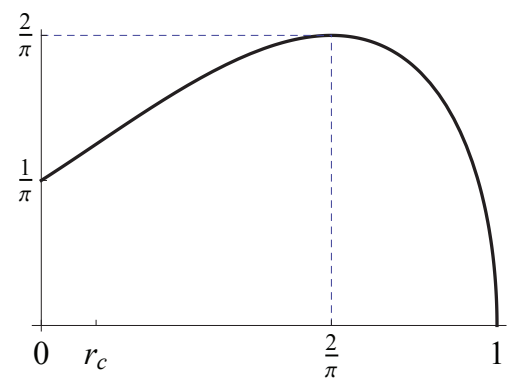

Figure 3 . The profile function $h$.

We call any set of the form $\left\{[z, t] \in \mathbb{H}^{n}: t^{-} \leq t \leq t^{+}\right\}$for some $t^{-} \leq t^{+}$a vertical segment. Given $p, q \in \mathbb{H}^{n}$ with $z_{p}=z_{q}$, we denote by $L_{p, q}$ the vertical segment joining $p$ to $q$,

$$
L_{p, q}=\left\{\left[z_{p}, t\right] \in \mathbb{H}^{n}: \min \left(t_{p}, t_{q}\right) \leq t \leq \max \left(t_{p}, t_{q}\right)\right\} .
$$

In the next proposition we state, for later use, an elementary geometric property of balls in $\mathbb{H}^{n}$. When not specified, by ball we mean a ball that can be either open or closed.

Proposition 2.3. The following statements hold:

(i) Let $B$ denote a ball in $\mathbb{H}^{n}$. For any $p, q \in B$ such that $z_{p}=z_{q}$, we have $L_{p, q} \subset B$.

(ii) For any $p \in \mathbb{H}^{n}$ and any $p_{1}, p_{2} \in \mathbb{H}^{n}$ such that $z_{p_{1}}=z_{p_{2}}$, we have

$$
d(p, q) \leq \max \left(d\left(p, p_{1}\right), d\left(p, p_{2}\right)\right)
$$

for all $q \in L_{p_{1}, p_{2}}$.

Proof. Property (i) holds for the (closed or open) unit ball by (2.5). Then this property follows for any ball using dilations and translations and noting that these maps are bijective maps that send vertical segments onto vertical segments.

To prove property (ii) set $r=\max \left(d\left(p, p_{1}\right), d\left(p, p_{2}\right)\right)$. We have $p_{1}, p_{2} \in \bar{B}(p, r)$. Hence $L_{p_{1}, p_{2}} \subset \bar{B}(p, r)$ by (i) and thus (ii) follows.

In the next lemma we deal with an outer vertical cone property for balls centered at the origin. Its proof (that we provide for the reader's convenience) follows from the local Lipschitz continuity of the profile function $h$ on $[0,1)$.

Lemma 2.4. Let $d>0$ and $\delta>0$ be fixed. There exists $\alpha(d, \delta)>0$ such that the following holds. If $p \in \partial B(0, d)$ is such that $t_{p} \geq \delta$, respectively $t_{p} \leq-\delta$, and $[w, s] \in \mathbb{H}^{n}$ is such that

$$
s>t_{p}+\alpha(d, \delta)\left\|w-z_{p}\right\|, \text { respectively } s<t_{p}-\alpha(d, \delta)\left\|w-z_{p}\right\|,
$$

then $[w, s] \notin \bar{B}(0, d)$. 
Proof. Let $\alpha=\alpha(d, \delta)$ be a positive constant to be chosen later. Let us consider the case where $p \in \partial B(0, d)$ and $[w, s] \in \mathbb{H}^{n}$ are such that $t_{p} \geq \delta$ and $s>t_{p}+\alpha\left\|w-z_{p}\right\|$, the other case being analogous. If $\|w\|>d$ then we obviously have $[w, s] \notin \bar{B}(0, d)$. If $\|w\| \leq d$ we want to prove that $s>h_{d}(\|w\|)$. We have

$$
s>t_{p}+\alpha\left\|w-z_{p}\right\|=h_{d}\left(\left\|z_{p}\right\|\right)+\alpha\left\|w-z_{p}\right\|,
$$

and thus it will be sufficient to show that

$$
h_{d}\left(\left\|z_{p}\right\|\right)+\alpha\left\|w-z_{p}\right\| \geq h_{d}(\|w\|) .
$$

Since $\lim _{r \rightarrow d^{-}} h_{d}(r)=0$, one can find $\bar{r}=\bar{r}(d, \delta) \in(0, d)$ such that $h_{d}(r)<\delta$ for all $r \in(\bar{r}, d]$. In particular $\left\|z_{p}\right\| \leq \bar{r}$ because $h_{d}\left(\left\|z_{p}\right\|\right)=t_{p} \geq \delta$. We choose $\alpha>0$ to be the Lipschitz constant of $h_{d}$ on $[0, \bar{r}]$. If $\|w\| \leq \bar{r}$ it follows

$$
h_{d}(\|w\|) \leq h_{d}\left(\left\|z_{p}\right\|\right)+\alpha\left|\|w\|-\left\|z_{p}\right\|\right| \leq h_{d}\left(\left\|z_{p}\right\|\right)+\alpha\left\|w-z_{p}\right\|
$$

as wanted. Whereas

$$
h_{d}(\|w\|)<\delta \leq h_{d}\left(\left\|z_{p}\right\|\right) \leq h_{d}\left(\left\|z_{p}\right\|\right)+\alpha\left\|w-z_{p}\right\|
$$

if $\|w\| \in(\bar{r}, d]$, which concludes the proof.

Remark 2.5. We note that, for any fixed $d>0$, the function $\alpha(d, \delta)$ can be taken to be continuous with respect to the variable $\delta$. This follows from the definition of $\alpha(d, \delta)=\left\|h_{d}^{\prime}\right\|_{L^{\infty}([0, \bar{r}(d, \delta)])}$ together with the fact that $\delta \mapsto \bar{r}(d, \delta)$ can be chosen to be continuous and the fact that the map $r \in(0, d) \mapsto\left\|h_{d}^{\prime}\right\|_{L^{\infty}([0, r])}$ is continuous.

\subsection{Two geometric transformations in $\mathbb{H}^{n}$}

In this subsection we introduce two geometric transformations, namely convexification along the vertical $t$-axis and Steiner symmetrization with respect to the $\mathbb{C}^{n}$-plane. They will play a crucial role in the sequel.

Given $F \subset \mathbb{H}^{n}$ we define its $t$-convex hull $t$-co $F$ by

$$
t \text {-co } F=\left\{p \in \mathbb{H}^{n}: p \in L_{p_{1}, p_{2}} \text { for some } p_{1}, p_{2} \in F \text { with } z_{p_{1}}=z_{p_{2}}\right\} \text {. }
$$

We say that $F$ is $t$-convex if $F=t$-co $F$.

Lemma 2.6. Let $F \subset \mathbb{H}^{n}$. We have $F \subset t$-co $F$ and $\operatorname{diam}(t$-co $F)=\operatorname{diam} F$.

Proof. We obviously have $F \subset t$-co $F$ and hence $\operatorname{diam} F \leq \operatorname{diam}(t$-co $F)$. Conversely, let $p, p^{\prime} \in t$-co $F$. One can find $p_{1}, p_{2} \in F$ with $z_{p_{1}}=z_{p_{2}}$ such that $p \in L_{p_{1}, p_{2}}$. Then it follows from Proposition 2.3(ii) that

$$
d\left(p^{\prime}, p\right) \leq \max \left(d\left(p^{\prime}, p_{1}\right), d\left(p^{\prime}, p_{2}\right)\right) .
$$

Similarly one can find $p_{1}^{\prime}, p_{2}^{\prime} \in F$ with $z_{p_{1}^{\prime}}=z_{p_{2}^{\prime}}$ such that $p^{\prime} \in L_{p_{1}^{\prime}, p_{2}^{\prime}}$. Then it follows once again from Proposition 2.3(ii) that

$$
\max \left(d\left(p^{\prime}, p_{1}\right), d\left(p^{\prime}, p_{2}\right)\right) \leq \max _{i, j=1,2} d\left(p_{i}, p_{j}^{\prime}\right) \leq \operatorname{diam} F,
$$

which concludes the proof. 
Given $F \subset \mathbb{H}^{n}$ measurable, its Steiner symmetrization St $F$ with respect to the $\mathbb{C}^{n}$-plane is defined by

$$
\text { St } F=\left\{[z, t] \in \mathbb{H}^{n}: z \in \pi(F), 2|t| \leq \mathcal{L}^{1}(\{s \in \mathbb{R}:[z, s] \in F\})\right\}
$$

where $\mathcal{L}^{1}$ denotes the one-dimensional Lebesgue measure and $\pi: \mathbb{H}^{n} \rightarrow \mathbb{C}^{n}$ is the canonical projection defined in (2.1). We define the reflection map $\sigma: \mathbb{H}^{n} \rightarrow \mathbb{H}^{n}$ as

$$
\sigma([z, t])=[\bar{z}, t]
$$

For the sake of simplicity, the following lemma is stated for compact sets. This will be the only case needed in this paper. It can however be easily generalized to noncompact sets.

Lemma 2.7. Let $F \subset \mathbb{H}^{n}$ be compact and such that $\sigma(F)=F$. Then $\operatorname{diam}(\operatorname{St} F) \leq$ $\operatorname{diam} F$.

Proof. Since $F$ is a compact subset of $\mathbb{H}^{n}$, then $t$-co $F$ is compact and is obviously $t$-convex. We have $\sigma(t$-co $F)=t$-co $F$ as soon as $\sigma(F)=F$. Since $\operatorname{St} F \subset \operatorname{St}(t$-co $F)$ and $\operatorname{diam}(t-\operatorname{co} F)=\operatorname{diam} F$ by Lemma 2.6, it is thus sufficient to consider $t$-convex compact sets $F$ such that $\sigma(F)=F$. Then we can describe $F$ as

$$
F=\left\{[z, t] \in \mathbb{H}^{n}: z \in \pi(F), a(z)-\mathcal{L}(z) \leq t \leq a(z)+\mathcal{L}(z)\right\}
$$

for some map $a: \pi(F) \rightarrow \mathbb{R}$ which satisfies $a(\bar{z})=a(z)$ for all $z \in \pi(F)$ and where $\mathcal{L}(z)=\mathcal{L}^{1}(\{s \in \mathbb{R}:[z, s] \in F\}) / 2$. Note that we also have $\mathcal{L}(\bar{z})=\mathcal{L}(z)$ for any $z \in \pi(F)$.

Let $p_{1}=\left[z_{1}, t_{1}\right] \in \mathrm{St} F$ and $p_{2}=\left[z_{2}, t_{2}\right] \in \mathrm{St} F$. Set

$$
\begin{aligned}
& F_{1}=\left\{[z, t] \in F: z=z_{1} \text { or } z=\bar{z}_{1}\right\}, \\
& F_{2}=\left\{[z, t] \in F: z=z_{2} \text { or } z=\bar{z}_{2}\right\} .
\end{aligned}
$$

We will prove that

$$
d\left(p_{1}, p_{2}\right) \leq \max \left(d\left(q_{1}, q_{2}\right): q_{1} \in F_{1}, q_{2} \in F_{2}\right) .
$$

Since $F_{1} \cup F_{2} \subset F$ this will imply $d\left(p_{1}, p_{2}\right) \leq \operatorname{diam} F$ as wanted.

Set $\hat{F}_{i}=\left[0,-a\left(z_{1}\right)\right] \cdot F_{i}, i=1,2$, i.e.,

$$
\hat{F}_{1}=\left\{z_{1}, \bar{z}_{1}\right\} \times\left[-\mathcal{L}\left(z_{1}\right), \mathcal{L}\left(z_{1}\right)\right] \quad \text { and } \quad \hat{F}_{2}=\left\{z_{2}, \bar{z}_{2}\right\} \times\left[b-\mathcal{L}\left(z_{2}\right), b+\mathcal{L}\left(z_{2}\right)\right],
$$

where $b=a\left(z_{2}\right)-a\left(z_{1}\right)$. The distance being left invariant, we have

$$
\max \left(d\left(q_{1}, q_{2}\right): q_{1} \in F_{1}, q_{2} \in F_{2}\right)=\max \left(d\left(q_{1}, q_{2}\right): q_{1} \in \hat{F}_{1}, q_{2} \in \hat{F}_{2}\right) .
$$

Next set $T_{2}=|b|+\mathcal{L}\left(z_{2}\right)$. We have $p_{2} \in\left\{z_{2}\right\} \times\left[-T_{2}, T_{2}\right]$ hence it follows from Proposition 2.3(ii) that

$$
d\left(p_{1}, p_{2}\right) \leq \max \left(d\left(p_{1},\left[z_{2}, T_{2}\right]\right), d\left(p_{1},\left[z_{2},-T_{2}\right]\right)\right) .
$$


Assume that $b \geq 0$. Then $\left[z_{2}, T_{2}\right] \in \hat{F}_{2}$ and since $p_{1} \in \hat{F}_{1}$ we get

$$
d\left(p_{1},\left[z_{2}, T_{2}\right]\right) \leq \max \left(d\left(q_{1}, q_{2}\right): q_{1} \in \hat{F}_{1}, q_{2} \in \hat{F}_{2}\right) .
$$

Let $\iota$ denote the isometry in $\left(\mathbb{H}^{n}, d\right)$ defined by

$$
\iota([z, t])=[\bar{z},-t] .
$$

We have $\iota\left(\hat{F}_{1}\right)=\hat{F}_{1}$ and $\iota\left(\left[z_{2},-T_{2}\right]\right)=\left[\bar{z}_{2}, T_{2}\right] \in \hat{F}_{2}$, hence

$$
d\left(p_{1},\left[z_{2},-T_{2}\right]\right)=d\left(\iota\left(p_{1}\right), \iota\left(\left[z_{2},-T_{2}\right]\right)\right) \leq \max \left(d\left(q_{1}, q_{2}\right): q_{1} \in \hat{F}_{1}, q_{2} \in \hat{F}_{2}\right) .
$$

Inequalities (2.10), (2.11) and (2.13) together with (2.9) give (2.8). The case where $b \leq 0$ can be treated in a similar way and this concludes the proof.

\section{Isodiametric problem}

We recall the definitions of the isodiametric constant

$$
C_{I}=\sup \left\{\mathcal{L}^{2 n+1}(F) /(\operatorname{diam} F)^{2 n+2}: 0<\operatorname{diam} F<+\infty\right\}
$$

and of the class of compact isodiametric sets

$$
\mathcal{I}=\left\{E \subset \mathbb{H}^{n}: E \text { compact, } \operatorname{diam} E>0, \mathcal{L}^{2 n+1}(E)=C_{I}(\operatorname{diam} E)^{2 n+2}\right\} .
$$

Recall that it is not restrictive to ask isodiametric sets to be compact as the closure of any set which realizes the supremum in the right-hand side of the definition of $C_{I}$ is a compact set that still realizes the supremum.

We also introduce the class of so-called rotationally invariant sets. Given $\theta=$ $\left(\theta_{1}, \ldots, \theta_{n}\right) \in \mathbb{R}^{n}$, we define the rotation $r_{\theta}: \mathbb{H}^{n} \rightarrow \mathbb{H}^{n}$ around the $\mathbb{R}$-axis by

$$
r_{\theta}([z, t])=\left[\left(e^{i \theta_{1}} z_{1}, \ldots, e^{i \theta_{n}} z_{n}\right), t\right] .
$$

Any such $r_{\theta}$ is an isometry in $\left(\mathbb{H}^{n}, d\right)$. We denote by $\mathcal{R}$ the class of rotationally invariant sets,

We set

$$
\mathcal{R}=\left\{F \subset \mathbb{H}^{n}: r_{\theta}(F) \subset F \text { for all } \theta \in \mathbb{R}^{n}\right\}
$$

$$
C_{I, \mathcal{R}}=\sup \left\{\mathcal{L}^{2 n+1}(F) /(\operatorname{diam} F)^{2 n+2}: F \in \mathcal{R}, 0<\operatorname{diam} F<+\infty\right\}
$$

and denote by $\mathcal{I}_{\mathcal{R}}$ the family of compact rotationally invariant sets that are isodiametric within the class of rotationally invariant sets,

$$
\mathcal{I}_{\mathcal{R}}=\left\{E \in \mathcal{R}: E \text { compact, } \operatorname{diam} E>0, \mathcal{L}^{2 n+1}(E)=C_{I, \mathcal{R}}(\operatorname{diam} E)^{2 n+2}\right\} .
$$

In other words, $\mathcal{I}$, resp. $\mathcal{I}_{\mathcal{R}}$, denotes the class of compact sets, resp. compact sets in $\mathcal{R}$, that maximize the $\mathcal{L}^{2 n+1}$-measure among all subsets of $\mathbb{H}^{n}$, resp. among all sets in $\mathcal{R}$, with the same diameter.

We first prove the existence of sets in $\mathcal{I}$ and $\mathcal{I}_{\mathcal{R}}$.

Theorem 3.1. Both families $\mathcal{I}$ and $\mathcal{I}_{\mathcal{R}}$ are nonempty. 
Proof. The proof that $\mathcal{I}$ is not empty relies on the compactness of equibounded sequences of nonempty compact sets with respect to the Hausdorff metric (see 2.10.21 in [8]) together with the upper semicontinuity of the Lebesgue measure (see Theorem 3.2 in [3]). The fact that $\mathcal{I}_{\mathcal{R}} \neq \emptyset$ as well can be proved in a similar way noting that $\mathcal{R}$ is closed with respect to the convergence of sets in the Hausdorff metric.

The rest of this section is devoted to the study of the regularity of sets in $\mathcal{I}$ and $\mathcal{I}_{\mathcal{R}}$. The necessary condition (NC) introduced in Section 1 will be one of the key ingredients in this study and we prove in the next proposition that sets in $\mathcal{I}$ and $\mathcal{I}_{\mathcal{R}}$ do satisfy this condition.

Proposition 3.2. Let $E \in \mathcal{I} \cup \mathcal{I}_{\mathcal{R}}$. Then $E$ satisfies the necessary condition (NC).

Proof. We argue by contradiction. Assume that $E \in \mathcal{I}$ and $p \in \partial E$ is such that $d(p, q)<\operatorname{diam} E$ for all $q \in \partial E$. The distance from $p$ being a continuous and open map and $E$ being compact, we have $\max _{q \in E} d(p, q)=\max _{q \in \partial E} d(p, q)$ (see Lemma 2.1 and Remark 2.2) and hence

$$
\max _{q \in E} d(p, q)<\operatorname{diam} E .
$$

Choosing $r=\left(\operatorname{diam} E-\max _{q \in E} d(p, q)\right) / 2>0$, it follows that

$$
\operatorname{diam}(E \cup \bar{B}(p, r))=\operatorname{diam} E .
$$

On the other hand, since $E$ is closed and $p \in \partial E$, we have $\operatorname{int}(B(p, r) \backslash E) \neq \emptyset$. This implies in particular that

$$
\mathcal{L}^{2 n+1}(E \cup \bar{B}(p, r))>\mathcal{L}^{2 n+1}(E)
$$

which contradicts the fact that $E \in \mathcal{I}$.

When $E \in \mathcal{I}_{\mathcal{R}}$ we modify the argument as follows. We set

$$
F=E \cup \bigcup_{\theta \in \mathbb{R}^{n}} \bar{B}\left(r_{\theta}(p), r\right)
$$

where $r=\left(\operatorname{diam} E-\max _{q \in E} d(p, q)\right) / 2>0$ as before. We have $F \in \mathcal{R}$ and

$$
\operatorname{diam} F=\operatorname{diam} E \text {. }
$$

Indeed, to prove (3.1) we fix $q_{1}, q_{2} \in F$. If $q_{1}, q_{2} \in E$ then $d\left(q_{1}, q_{2}\right) \leq \operatorname{diam} E$. If $q_{1}, q_{2} \in F \backslash E$ then there exist $\theta_{1}, \theta_{2} \in \mathbb{R}^{n}$ such that $d\left(q_{i}, r_{\theta_{i}}(p)\right) \leq r, i=1,2$. Recalling that any rotation $r_{\theta}$ is an isometry in $\left(\mathbb{H}^{n}, d\right)$ and that $E \in \mathcal{R}$, it follows that

$$
\begin{aligned}
d\left(q_{1}, q_{2}\right) & \leq d\left(r_{\theta_{1}}(p), r_{\theta_{2}}(p)\right)+2 r=d\left(p, r_{\theta_{1}-\theta_{2}}(p)\right)+2 r \\
& \leq \max _{q \in E} d(p, q)+2 r=\operatorname{diam} E .
\end{aligned}
$$

If $q_{1} \in E$ and $q_{2} \in F \backslash E$ with $d\left(q_{2}, r_{\theta_{2}}(p)\right) \leq r$ for some $\theta_{2} \in \mathbb{R}^{n}$, we have

$$
d\left(q_{1}, q_{2}\right) \leq d\left(q_{1}, r_{\theta_{2}}(p)\right)+r \leq d\left(p, r_{-\theta_{2}}\left(q_{1}\right)\right)+r \leq \max _{q \in E} d(p, q)+r \leq \operatorname{diam} E .
$$


On the other hand, similarly as before, we have

$$
\mathcal{L}^{2 n+1}(F)>\mathcal{L}^{2 n+1}(E)
$$

and this contradicts the fact that $E \in \mathcal{I}_{\mathcal{R}}$.

Let us introduce some notation. Given a compact set $E$, we define $f^{+}, f^{-}, U$ and $\hat{E}$ as follows. We set

$$
\begin{aligned}
& f^{+}(z)=\max (t \in \mathbb{R}:[z, t] \in E), \\
& f^{-}(z)=\min (t \in \mathbb{R}:[z, t] \in E)
\end{aligned}
$$

for all $z \in \pi(E)$. Clearly, $\left[z, f^{ \pm}(z)\right] \in \partial E$ for all $z \in \pi(E)$. Recalling the definition of $t$-co $E$ given in Subsection 2.4, one has

$$
t \text {-co } E=\left\{[z, t] \in \mathbb{H}^{n}: z \in \pi(E), f^{-}(z) \leq t \leq f^{+}(z)\right\},
$$

and $t$-co $E$ is itself compact.

We set

$$
U=\left\{z \in \pi(E): f^{-}(z)<f^{+}(z)\right\}
$$

and

$$
\hat{E}=\left\{[z, t] \in \mathbb{H}^{n}: z \in \bar{U}, f^{-}(z) \leq t \leq f^{+}(z)\right\} .
$$

Since $\pi(E)$ is closed, we have $\bar{U} \subset \pi(E)$. In particular $f^{+}$and $f^{-}$are well defined on $\bar{U}$. Moreover $\hat{E}=t$-co $E \cap \pi^{-1}(\bar{U})$ is compact and contained in $t$-co $E$.

We are now ready to state our key regularity result. It concerns $t$-convex and compact sets satisfying (NC).

Theorem 3.3. For any t-convex and compact set $E$ satisfying (NC) the following properties hold.

(i) The set $U$ is open in $\mathbb{C}^{n}$ and the maps $f^{-}$and $f^{+}$are locally Lipschitz on $U$ and continuous on $\pi(E)$.

(ii) $\mathcal{L}^{2 n+1}(E)=\mathcal{L}^{2 n+1}(\hat{E})$.

(iii) We have

$$
\begin{aligned}
\operatorname{int} E=\operatorname{int} \hat{E} & =\left\{[z, t] \in \mathbb{H}^{n}: z \in U, f^{-}(z)<t<f^{+}(z)\right\}, \\
\partial \hat{E} & =\left\{\left[z, f^{ \pm}(z)\right] \in \mathbb{H}^{n}: z \in \bar{U}\right\}, \\
\hat{E} & =\overline{\operatorname{int} E} .
\end{aligned}
$$

Before proving Theorem 3.3, we introduce some notation and give a technical lemma.

Given $r>0$ and $p_{1}, p_{2} \in \mathbb{H}^{n}$ with $\pi\left(p_{1}\right)=\pi\left(p_{2}\right), p_{1}=\left[z_{12}, t_{1}\right]$ and $p_{2}=\left[z_{12}, t_{2}\right]$ for some $z_{12} \in \mathbb{C}^{n}$ and $t_{1}, t_{2} \in \mathbb{R}$, we set $\delta_{12}=\left(t_{2}-t_{1}\right) / 2$ and

$$
F_{p_{1}, p_{2}}(r)=\left\{[w, s] \in \mathbb{H}^{n}:\left\|w-z_{12}\right\| \leq r,\left|s-\frac{t_{1}+t_{2}}{2}\right| \leq \delta_{12}\left(1-\frac{\left\|w-z_{12}\right\|}{r}\right)\right\}
$$

whenever $\delta_{12}>0$. Clearly, $F_{p_{1}, p_{2}}\left(r_{1}\right) \subset F_{p_{1}, p_{2}}\left(r_{2}\right)$ provided $r_{1} \leq r_{2}$. 
Lemma 3.4. Let $C>0, d>0$ and $\delta>0$ be fixed. There exists $\gamma(C, d, \delta)>0$ such that the following holds: For any $r \in(0, \gamma(C, d, \delta)], p_{1}=\left[z_{12}, t_{1}\right]$ and $p_{2}=$ $\left[z_{12}, t_{2}\right] \in \mathbb{H}^{n}$ such that $\left\|z_{12}\right\| \leq C$ and $\delta_{12}=\delta$, we have

$$
F_{p_{1}, p_{2}}(r) \subset \bar{B}(p, d)
$$

for all $p \in \mathbb{H}^{n}$ such that $p_{1}, p_{2} \in \bar{B}(p, d)$.

Proof. Set $p_{0}=\left[-z_{12},-\left(t_{1}+t_{2}\right) / 2\right]$. After a left translation by $p_{0}$ we need to prove that for all $r>0$ small enough we have

$$
p_{0} \cdot F_{p_{1}, p_{2}}(r) \subset \bar{B}(p, d)
$$

for all $p \in \mathbb{H}^{n}$ such that $d\left(p, p_{0} \cdot p_{1}\right) \leq d$ and $d\left(p, p_{0} \cdot p_{2}\right) \leq d$. For such a $p=[z, t] \in \mathbb{H}^{n}$ we have $\left(p_{0} \cdot p_{i}\right)^{-1} \cdot p=[z, t \pm \delta] \in \bar{B}(0, d)$ for $i=1,2$, hence $\|z\| \leq d$ and $|t \pm \delta| \leq h_{d}(\|z\|)$. It follows that $h_{d}(\|z\|) \geq|t|+\delta \geq \delta$. Recalling that $\lim _{u \rightarrow d^{-}} h_{d}(u)=0$ and considering $\bar{r}=\bar{r}(d, \delta) \in(d / 2, d)$ such that $h_{d}(u)<\delta$ whenever $u \in(\bar{r}, d]$, we get that $\|z\| \leq \bar{r}$.

We have

$$
p_{0} \cdot F_{p_{1}, p_{2}}(r)=\left\{[w, s] \in \mathbb{H}^{n}:\|w\| \leq r,\left|s+2 \operatorname{Im} z_{12} \bar{w}\right| \leq \delta\left(1-\frac{\|w\|}{r}\right)\right\} .
$$

Let $[w, s] \in p_{0} \cdot F_{p_{1}, p_{2}}(r)$ and let us show that $p^{-1} \cdot[w, s] \in \bar{B}(0, d)$ or equivalently that $\|w-z\| \leq d$ and

$$
|s-t-2 \operatorname{Im} z \bar{w}| \leq h_{d}(\|w-z\|)
$$

provided $r>0$ is small enough. First note that $\|w-z\| \leq r+\bar{r}$ is less than $d$ provided $r \leq d-\bar{r}$.

Next we have

$$
\begin{aligned}
s-t-2 \operatorname{Im} z \bar{w} & \leq \delta\left(1-\frac{\|w\|}{r}\right)-2 \operatorname{Im} z_{12} \bar{w}-t-2 \operatorname{Im} z \bar{w} \\
& \leq(\delta-t)-\frac{\delta}{r}\|w\|+2\left\|z_{12}\right\|\|w\|+2\|z\|\|w\| \\
& \leq h_{d}(\|z\|)-\left(\frac{\delta}{r}-(2 C+2 d)\right)\|w\|
\end{aligned}
$$

where the last inequality follows from the fact that $[-z, \delta-t]=[z, t-\delta]^{-1} \in \bar{B}(0, d)$. On the other hand if $r \leq(d-\bar{r}) / 2$, we have $\max (\|w-z\|,\|z\|) \leq(d+\bar{r}) / 2<d$. Let $M=M(d, \delta)>0$ denote the Lipschitz constant of $h_{d}$ on $[0,(d+\bar{r}) / 2]$. Then we have

$$
h_{d}(\|z\|) \leq h_{d}(\|w-z\|)+M\|w\|
$$

It follows that

$$
s-t-2 \operatorname{Im} z \bar{w} \leq h_{d}(\|w-z\|)-\left(\frac{\delta}{r}-(2 C+2 d+M)\right)\|w\| \leq h_{d}(\|w-z\|)
$$

provided $r \leq \min ((d-\bar{r}) / 2, \delta /(2 C+2 d+M))$. 
Similarly we have

$$
\begin{aligned}
s-t-2 \operatorname{Im} z \bar{w} & \geq-\delta\left(1-\frac{\|w\|}{r}\right)-2 \operatorname{Im} z_{12} \bar{w}-t-2 \operatorname{Im} z \bar{w} \\
& \geq-h_{d}(\|z\|)+\left(\frac{\delta}{r}-(2 C+2 d)\right)\|w\| \\
& \geq-h_{d}(\|w-z\|)+\left(\frac{\delta}{r}-(2 C+2 d+M)\right)\|w\| \\
& \geq-h_{d}(\|w-z\|)
\end{aligned}
$$

provided $r \leq \min ((d-\bar{r}) / 2, \delta /(2 C+2 d+M))$ and where the second inequality follows from the fact that $[-z,-t-\delta]=[z, t+\delta]^{-1} \in \bar{B}(0, d)$. Hence the lemma follows with

$$
\gamma(C, d, \delta)=\min \left(\frac{d-\bar{r}(d, \delta)}{2}, \frac{\delta}{2 C+2 d+M(d, \delta)}\right)
$$

Remark 3.5. Note that, $C$ and $d$ being fixed, the function $\gamma(C, d, \delta)$ can be taken to be continuous with respect to the variable $\delta$. This is a consequence of (3.2) and the fact that $\bar{r}(d, \delta)$ can be chosen continuous with respect to $\delta$.

We turn now to the proof of Theorem 3.3.

Proof of Theorem 3.3. We fix a $t$-convex and compact set $E$ satisfying (NC) and set

$$
C=\max _{p \in E}\|\pi(p)\| \quad \text { and } \quad d=\operatorname{diam} E .
$$

We begin with two lemmata. The first one is a consequence of Lemma 3.4.

Lemma 3.6. Let $E$ be as above, let $\delta>0$ be fixed and let $\gamma=\gamma(C, d, \delta)>0$ be as in Lemma 3.4. Then, for any $r \in(0, \gamma]$, one has

$$
F_{p_{1}, p_{2}}(r) \subset E
$$

for all $p_{1}, p_{2} \in E$ such that $\pi\left(p_{1}\right)=\pi\left(p_{2}\right)$ and $\delta_{12}=\delta$.

Proof. Since $E$ is closed, it is enough to show that

$$
\operatorname{int} F_{p_{1}, p_{2}}(r) \subset E \text {. }
$$

Assume by contradiction that (int $\left.F_{p_{1}, p_{2}}(r)\right) \backslash E$ is nonempty. By $t$-convexity of $E$ we know that $E \cap \operatorname{int} F_{p_{1}, p_{2}}(r)$ is also nonempty since it contains $L_{p_{1}, p_{2}} \backslash\left\{p_{1}, p_{2}\right\}$. Therefore there exists $p \in \partial E \cap \operatorname{int} F_{p_{1}, p_{2}}(r)$. Then by (NC) there exists $q \in \partial E$ such that $d(p, q)=d$. Thanks to Lemma 3.4, we have

$$
p \in \operatorname{int} F_{p_{1}, p_{2}}(r) \subset B(q, d) .
$$

Thus $d(p, q)<d$, which is a contradiction. 
Lemma 3.7. Let $E$ be as before and let $\delta>0$ be fixed. There exists $\beta(C, d, \delta)>0$ such that the following holds: If $z \in U$ is such that $f^{+}(z)-f^{-}(z)=2 \delta$, then

$$
f^{+}(w) \leq f^{+}(z)+\beta(C, d, \delta)\|w-z\| \quad \text { and } \quad f^{-}(w) \geq f^{-}(z)-\beta(C, d, \delta)\|w-z\|
$$

for all $w \in \pi(E)$.

Proof. We claim that the lemma holds for $\beta(C, d, \delta)=\alpha(d, \delta)+2 C$, where $\alpha(d, \delta)$ is given by Lemma 2.4. Assume by contradiction that $w \in \pi(E)$ is such that

$$
f^{+}(w)>f^{+}(z)+(\alpha(d, \delta)+2 C)\|w-z\|,
$$

the case where $f^{-}(w)<f^{-}(z)-(\alpha(d, \delta)+2 C)\|w-z\|$ being analogous. Since $\left[z, f^{+}(z)\right] \in \partial E$, by $(\mathrm{NC})$ there exists $q \in \partial E$ such that $d\left(\left[z, f^{+}(z)\right], q\right)=d$. Set $p=q^{-1} \cdot\left[z, f^{+}(z)\right]$ and $\left[w^{\prime}, s^{\prime}\right]=q^{-1} \cdot\left[w, f^{+}(w)\right]$. We have

$$
p=\left[z-z_{q}, f^{+}(z)-t_{q}-2 \operatorname{Im} z_{q} \bar{z}\right]
$$

and

It follows that

$$
\left[w^{\prime}, s^{\prime}\right]=\left[w-z_{q}, f^{+}(w)-t_{q}-2 \operatorname{Im} z_{q} \bar{w}\right]
$$

$$
\begin{aligned}
s^{\prime} & =t_{p}+f^{+}(w)-f^{+}(z)-2 \operatorname{Im} z_{q}(\overline{w-z}) \\
& >t_{p}+(\alpha(d, \delta)+2 C)\|w-z\|-2 C\|w-z\|=t_{p}+\alpha(d, \delta)\left\|w^{\prime}-z_{p}\right\|,
\end{aligned}
$$

where the inequality follows by the choice of $w \in \pi(E)$.

On the other hand set $p^{\prime}=q^{-1} \cdot\left[z, f^{-}(z)\right]$. We have $z_{p^{\prime}}=z_{p}$. We also have $\left[z, f^{-}(z)\right] \in E$ and $q \in E$, hence $d\left(q,\left[z, f^{-}(z)\right]\right) \leq d$ or equivalently $p^{\prime} \in \bar{B}(0, d)$. Therefore,

$$
\left|t_{p^{\prime}}\right| \leq h_{d}\left(\left\|z_{p}\right\|\right)
$$

Recalling that $p \in \partial B(0, d)$ we get

$$
t_{p}=h_{d}\left(\left\|z_{p}\right\|\right) \geq \frac{t_{p}-t_{p^{\prime}}}{2}=\frac{f^{+}(z)-f^{-}(z)}{2}=\delta .
$$

Taking Lemma 2.4 into account, we infer from (3.3) and (3.4) that $\left[w^{\prime}, s^{\prime}\right] \notin \bar{B}(0, d)$, i.e., $d\left(q,\left[w, f^{+}(w)\right]\right)>d$. This implies that diam $E>d$, a contradiction.

Remark 3.8. Taking into account Remark 2.5, one can take the function $\beta(C, d, \delta)$ to be continuous with respect to the variable $\delta$.

We prove now the continuity of $f^{-}$and $f^{+}$on $\pi(E)$.

Lemma 3.9. The functions $f^{-}$and $f^{+}$are continuous on $\pi(E)$.

Proof. Let $z \in \pi(E)$ and let us prove that $f^{+}$is continuous at $z$, the case of the function $f^{-}$being similar. Let $\left(z_{j}\right)$ be a sequence of points in $\pi(E)$ such that $z_{j} \rightarrow z$ as $j \rightarrow \infty$. Since $E$ is compact, $f^{+}$is bounded and to prove the continuity of $f^{+}$at $z$ it is sufficient to prove that any possible limit of the sequence $\left(f^{+}\left(z_{j}\right)\right)$ coincides with $f^{+}(z)$. By contradiction assume that $f^{+}\left(z_{j}\right) \rightarrow t$ as $j \rightarrow \infty$ for some $t \neq f^{+}(z)$. Since $E$ is compact, hence closed, and $\left[z_{j}, f^{+}\left(z_{j}\right)\right] \in E$, we have $[z, t] \in E$. It follows in particular that, by definition of $f^{+}$and $f^{-}$, we must have 
that $f^{+}(z)>t \geq f^{-}(z)$. Setting $p_{1}=\left[z, f^{-}(z)\right]$ and $p_{2}=\left[z, f^{+}(z)\right]$, we thus have $\delta_{12}=\left(f^{+}(z)-f^{-}(z)\right) / 2>0$. Owing to Lemma 3.6, one obtains that $F_{p_{1}, p_{2}}(\gamma) \subset E$, where $\gamma=\gamma\left(C, d, \delta_{12}\right)$ is given by Lemma 3.4. Therefore, recalling once again the definition of $f^{+}$, we get that $\liminf _{j \rightarrow \infty} f^{+}\left(z_{j}\right) \geq f^{+}(z)$, a contradiction.

We turn now to the proof of the fact that $U$ is open, and that $f^{-}$and $f^{+}$are locally Lipschitz continuous on $U$.

Lemma 3.10. The set $U \subset \mathbb{C}^{n}$ is open, and the maps $f^{-}$and $f^{+}$are locally Lipschitz continuous on $U$.

Proof. Let us introduce some notation. We will denote by $\mathscr{B}(z, r)$ the open ball in $\mathbb{C}^{n}$ with center $z \in \mathbb{C}^{n}$ and radius $r>0$. Given $z \in U$, we set

$$
\delta_{z}=\frac{f^{+}(z)-f^{-}(z)}{2}
$$

and

$$
\gamma_{z}=\gamma\left(C, d, \delta_{z}\right), \quad \beta_{z}=\beta\left(C, d, \delta_{z}\right)
$$

where $\gamma\left(C, d, \delta_{z}\right)$ and $\beta\left(C, d, \delta_{z}\right)$ are given, respectively, by Lemmas 3.4 and 3.7.

We first prove that $U \subset \mathbb{C}^{n}$ is open. Let $z \in U$. Set $t_{1}=f^{-}(z), t_{2}=f^{+}(z)$ and let $p_{1}=\left[z, t_{1}\right], p_{2}=\left[z, t_{2}\right] \in E$. It follows from Lemma 3.6 that $F_{p_{1}, p_{2}}\left(\gamma_{z}\right) \subset E$. In particular for any $w \in \mathbb{C}^{n}$ with $\|w-z\|<\gamma_{z}$, we have $[w, s] \in E$ for all

$$
\begin{aligned}
s \in & {\left[\frac{t_{1}+t_{2}}{2}-\delta_{z}\left(1-\frac{\|w-z\|}{\gamma_{z}}\right), \frac{t_{1}+t_{2}}{2}+\delta_{z}\left(1-\frac{\|w-z\|}{\gamma_{z}}\right)\right] } \\
& =\left[t_{1}+\delta_{z} \frac{\|w-z\|}{\gamma_{z}}, t_{2}-\delta_{z} \frac{\|w-z\|}{\gamma_{z}}\right] .
\end{aligned}
$$

Since

$$
t_{1}+\delta_{z} \frac{\|w-z\|}{\gamma_{z}}<t_{2}-\delta_{z} \frac{\|w-z\|}{\gamma_{z}}
$$

as soon as $\|w-z\|<\gamma_{z}$, it follows that $\mathscr{B}\left(z, \gamma_{z}\right) \subset U$. Hence $U \subset \mathbb{C}^{n}$ is open.

We prove now that $f^{-}$and $f^{+}$are locally Lipschitz continuous on $U$. Let $z \in U$. By the previous argument we already know that

$$
f^{+}(w) \geq f^{+}(z)-\delta_{z} \frac{\|w-z\|}{\gamma_{z}} \quad \text { and } \quad f^{-}(w) \leq f^{-}(z)+\delta_{z} \frac{\|w-z\|}{\gamma_{z}}
$$

for all $w \in \mathscr{B}\left(z, \gamma_{z}\right)$. Next, Lemma 3.7 implies that, for all $w \in \mathscr{B}\left(z, \gamma_{z}\right) \subset \pi(E)$,

$$
f^{+}(w) \leq f^{+}(z)+\beta_{z}\|w-z\| \quad \text { and } \quad f^{-}(w) \geq f^{-}(z)-\beta_{z}\|w-z\| .
$$

We fix a compact set $K \subset U$ and define $L=\sup _{z \in K} \max \left(\beta_{z}, \delta_{z} / \gamma_{z}\right)$ and $\gamma=\inf _{z \in K} \gamma_{z}$. Owing to the continuity of $\delta_{z}, \beta_{z}$ and $\gamma_{z}$ (see Lemma 3.9 and Remarks 3.5 and 3.8), one has $L<+\infty$ and $\gamma>0$. Therefore, for any $z, w \in K$ satisfying $\|z-w\|<\gamma$ we conclude that

$$
\left|f^{+}(w)-f^{+}(z)\right| \leq L\|w-z\| \quad \text { and } \quad\left|f^{-}(w)-f^{-}(z)\right| \leq L\|w-z\| .
$$

Hence $f^{+}$and $f^{-}$are Lipschitz continuous on $K$, as desired. 
We are now ready to conclude the proof of Theorem 3.3. Statement (i) follows from Lemmata 3.9 and 3.10. Statement (ii) is a consequence of

$$
E \backslash \hat{E}=\left\{[z, t] \in \mathbb{H}^{n}: z \in \pi(E) \backslash \bar{U}, t=f^{+}(z)\right\}
$$

and of the continuity of $f^{+}$. Finally (iii) is straightforward and follows from (i) by standard arguments.

We are going to apply Theorem 3.3 to sets in $\mathcal{I}$ and $\mathcal{I}_{\mathcal{R}}$. In order to do this, we first need to prove that such sets are $t$-convex.

Proposition 3.11. Any set $E \in \mathcal{I} \cup \mathcal{I}_{\mathcal{R}}$ is t-convex.

Proof. Assume by contradiction that one can find $p=[z, t] \in t$-co $E \backslash E$. By definition of $t$-co $E$, one must have $f^{-}(z)<t<f^{+}(z)$ and hence $z \in U$.

We have $E \subset t$-co $E$ and diam $t$-co $E=\operatorname{diam} E$ by Lemma 2.6. Since $t$-co $E \in \mathcal{R}$ whenever $E \in \mathcal{R}$, this implies that $t$-co $E \in \mathcal{I} \cup \mathcal{I}_{\mathcal{R}}$. Hence $t$-co $E$ is a compact set that satisfies (NC) (see Proposition 3.2) and is obviously $t$-convex. Then Theorem 3.3 applies to $t$-co $E$. Noting that the maps $f^{ \pm}$, and consequently the set $U$, associated to $E$ and $t$-co $E$ coincide, we get from Theorem 3.3 (iii) that $p \in \operatorname{int}(t$-co $E)$.

Since $E$ is closed and $p \notin E$ it follows that $p \in \operatorname{int}(t$-co $E \backslash E)$. In particular $\operatorname{int}(t$-co $E \backslash E) \neq \emptyset$ and $\mathcal{L}^{2 n+1}(t$-co $E \backslash E)>0$. Recalling that $E \subset t$-co $E$ and that both $E$ and $t$-co $E$ belong to $\mathcal{I}$, resp. $\mathcal{I}_{\mathcal{R}}$, with $\operatorname{diam}(t$-co $E)=\operatorname{diam} E$, this gives a contradiction.

Noting that $\hat{E} \subset E$ whenever $E$ is $t$-convex and that $\hat{E} \in \mathcal{R}$ whenever $E \in \mathcal{R}$, we get from Theorem 3.3(ii) that $\hat{E} \in \mathcal{I}$, resp. $\hat{E} \in \mathcal{I}_{\mathcal{R}}$, whenever $E \in \mathcal{I}$, resp. $E \in \mathcal{I}_{\mathcal{R}}$, with $\operatorname{diam} \hat{E}=\operatorname{diam} E$.

We gather in the next theorem the properties of sets in $\mathcal{I} \cup \mathcal{I}_{\mathcal{R}}$ proved in this section. Recall that the notations used in the statement to follow are those introduced before Theorem 3.3.

Theorem 3.12. For any set $E \in \mathcal{I} \cup \mathcal{I}_{\mathcal{R}}$, the following properties hold:

(i) $E$ is t-convex.

(ii) $\mathcal{L}^{2 n+1}(\hat{E})=\mathcal{L}^{2 n+1}(E)$ and $\operatorname{diam} \hat{E}=\operatorname{diam} E$.

(iii) $\hat{E} \in \mathcal{I}$, resp. $\hat{E} \in \mathcal{I}_{\mathcal{R}}$, whenever $E \in \mathcal{I}$, resp. $E \in \mathcal{I}_{\mathcal{R}}$.

(iv) The set $U$ is open in $\mathbb{C}^{n}$ and the maps $f^{-}$and $f^{+}$are locally Lipschitz on $U$ and continuous on $\pi(E)$.

(v) We have

$$
\begin{aligned}
\operatorname{int} E=\operatorname{int} \hat{E} & =\left\{[z, t] \in \mathbb{H}^{n}: z \in U, f^{-}(z)<t<f^{+}(z)\right\}, \\
\partial \hat{E} & =\left\{\left[z, f^{ \pm}(z)\right] \in \mathbb{H}^{n}: z \in \bar{U}\right\}, \\
\hat{E} & =\overline{\operatorname{int} E} .
\end{aligned}
$$




\section{Isodiametric problem for rotationally invariant sets}

In this section we characterize the Steiner symmetrization with respect to the $\mathbb{C}^{n}$ plane of sets $E \in \mathcal{I}_{\mathcal{R}}$. Our main result states that the set $S t E$ belongs to $\mathcal{I}_{\mathcal{R}}$ and is uniquely determined once the diameter of $E$ is fixed. It coincides with a particular set $A_{\text {diam } E}$, defined below, that consequently also belongs to $\mathcal{I}_{\mathcal{R}}$.

Constructing suitable perturbations of this set that preserve the diameter and the $\mathcal{L}^{2 n+1}$-measure, see Proposition 4.5, we also get two remarkable consequences. First, the essential non uniqueness of sets in $\mathcal{I}_{\mathcal{R}}$, see Corollary 4.6. Second, the existence of sets in $\mathcal{I}$ which are not rotationally invariant even up to isometries, see Corollary 4.7.

Given $\lambda>0$, we set

$$
l_{\lambda}(r)= \begin{cases}\frac{\lambda^{2}}{2 \pi} & \text { if } r \in[0, \lambda / \pi] \\ h_{\frac{\lambda}{2}}(r) & \text { if } r \in[\lambda / \pi, \lambda / 2]\end{cases}
$$

(see (2.6) for the definition of $h_{\frac{\lambda}{2}}$ ) and

$$
A_{\lambda}=\left\{[z, t] \in \mathbb{H}^{n}: 2\|z\| \leq \lambda,|t| \leq l_{\lambda}(\|z\|)\right\} .
$$

We have

$$
A_{\lambda}=A_{\lambda}^{1} \cup A_{\lambda}^{2}
$$

where

$$
A_{\lambda}^{1}=\left\{[z, t] \in \mathbb{H}^{n}:\|z\| \leq \lambda / \pi, 2 \pi|t| \leq \lambda^{2}\right\}
$$

and

$$
\begin{aligned}
A_{\lambda}^{2} & =\left\{[z, t] \in \mathbb{H}^{n}: \lambda / \pi \leq\|z\| \leq \lambda / 2,|t| \leq h_{\frac{\lambda}{2}}(r)\right\} \\
& =\bar{B}(0, \lambda / 2) \cap\left\{[z, t] \in \mathbb{H}^{n}:\|z\| \geq \lambda / \pi\right\} .
\end{aligned}
$$

The set $A_{\lambda}$ is the closed convex hull, in the Euclidean sense when identifying $\mathbb{H}^{n}$ with $\mathbb{R}^{2 n+1}$, of the ball $B(0, \lambda / 2)$ in $\left(\mathbb{H}^{n}, d\right)$ centered at the origin and with diameter $\lambda$. See Figure 1 in Section 1 for a picture.

We first show that the diameter of $A_{\lambda}$ equals $\lambda$.

Proposition 4.1. We have $\operatorname{diam} A_{\lambda}=\lambda$ for all $\lambda>0$.

Proof. Since $A_{\lambda}=\delta_{\lambda}\left(A_{1}\right)$ for all $\lambda>0$, it is enough to prove that $\operatorname{diam} A_{1}=1$. We begin with a technical lemma.

Lemma 4.2. Let $d>0, r \leq d / \pi$ and $t \in \mathbb{R}$ be fixed. Set

$$
C=\left\{[z, t] \in \mathbb{H}^{n}:\|z\|=r\right\} \quad \text { and } \quad D=\left\{[z, t] \in \mathbb{H}^{n}:\|z\| \leq r\right\} .
$$

Let $p \in \mathbb{H}^{n}$. Assume that $\operatorname{diam}(C \cup\{p\}) \leq d$ and $\operatorname{diam}(D \cup\{p\})>d$. Then $\left\|z_{p}\right\|<r$ and there exists $q \in D \backslash C$ such that $z_{p}=z_{q}$ and $\operatorname{diam}(D \cup\{p\})=d(p, q)$.

Proof. We have $d\left([z, t],\left[z^{\prime}, t\right]\right) \leq d([z, t],[0, t])+d\left([0, t],\left[z^{\prime}, t\right]\right)=\|z\|+\left\|z^{\prime}\right\| \leq 2 r$ for all $[z, t],\left[z^{\prime}, t\right] \in D$ and $d([z, t],[-z, t])=2\|z\|=2 r$ for all $[z, t] \in C$ (see $\left.(2.2)\right)$. It follows that $2 r=\operatorname{diam} C=\operatorname{diam} D \leq \operatorname{diam}(C \cup\{p\})<\operatorname{diam}(D \cup\{p\})$, and hence $\operatorname{diam}(D \cup\{p\})=d(p, q)$ for some $q=\left[z_{q}, t\right] \in D \backslash C$. 
Set $\bar{d}=\operatorname{diam}(D \cup\{p\})$ and

$$
h_{p, \bar{d}}^{ \pm}(z)= \pm h_{\bar{d}}\left(\left\|z-z_{p}\right\|\right)+t_{p}+2 \operatorname{Im} z_{p} \bar{z}
$$

so that

$$
\bar{B}(p, \bar{d})=\left\{[z, s] \in \mathbb{H}^{n}:\left\|z-z_{p}\right\| \leq \bar{d}, h_{p, \bar{d}}^{-}(z) \leq s \leq h_{p, \bar{d}}^{+}(z)\right\}
$$

We have $q=\left[z_{q}, t\right] \in \partial B(p, \bar{d})$. Changing if necessary $p, C$ and $D$ into $\iota(p), \iota(C)$ and $\iota(D)$ (see $(2.12)$ for the definition of $\iota$ ) one can assume with no loss of generality that $t=h_{p, \bar{d}}^{+}\left(z_{q}\right)$.

We have $D \subset \bar{B}(p, \bar{d})$. First we note that this implies that $\left\|z_{q}-z_{p}\right\|<\bar{d}$. Otherwise, since $\left\|z_{q}\right\|<r$ we could find $z$ close enough to $z_{q}$ such that $[z, t] \in D$ with $\left\|z-z_{p}\right\|>\bar{d}$. On the other hand we have $\pi(D) \subset \pi(\bar{B}(p, \bar{d}))=\left\{w \in \mathbb{C}^{n}\right.$ : $\left.\left\|w-z_{p}\right\| \leq \bar{d}\right\}$ which gives a contradiction. It follows that the map $h_{p, \bar{d}}^{+}$is well defined on an open neighbourhood of $z_{q}$ in $\mathbb{C}^{n}$. Next since $D \subset \bar{B}(p, \bar{d})$ and $q$ is in the relative interior of $D$ in $\mathbb{C}^{n} \times\{t\}$ it follows that $h_{p, \bar{d}}^{+}$admits a local minimum at $z_{q}$. Assume now by contradiction that $z_{q} \neq z_{p}$. Then $h_{p, \bar{d}}^{+}$is differentiable at $z_{q}$ and we get that

$$
\bar{d} h^{\prime}\left(\frac{\left\|z_{q}-z_{p}\right\|}{\bar{d}}\right) \frac{z_{q}-z_{p}}{\left\|z_{q}-z_{p}\right\|}-2 z_{p}^{\perp}=0
$$

where $z_{p}^{\perp}=i z_{p}$. This implies that $z_{q} \neq 0$. Indeed otherwise (4.1) implies that $z_{p}=0$ and hence $z_{q}=z_{p}$. Next if $z_{p} \neq 0$ we get from (4.1) that $z_{q}=z_{p}+\left\langle z_{q}, z_{p}^{\perp}\right\rangle z_{p}^{\perp}$ with $\left\langle z_{q}, z_{p}^{\perp}\right\rangle \neq 0$ where the scalar product is that of $\mathbb{R}^{2 n}$ after identifying points in $\mathbb{C}^{n}$ with points in $\mathbb{R}^{2 n}$. It follows that $\left\|z_{q}\right\|>\left\|z_{p}\right\|$ which also holds true if $z_{p}=0$. On the other hand, restricting to a segment $s \in(-\varepsilon, \varepsilon) \mapsto z_{q}+s\left(z_{q}-z_{p}\right)$ for $\varepsilon>0$ small enough we get that $h^{\prime \prime}\left(\left\|z_{q}-z_{p}\right\| / \bar{d}\right) \geq 0$ hence $\left\|z_{q}-z_{p}\right\| / \bar{d} \leq r_{c}$ where $\left[0, r_{c}\right]$ is the interval where $h^{\prime}$ is increasing (see Subsection 2.3). Since $\left\|z_{q}-z_{p}\right\|>0$ it follows that

$$
h^{\prime}\left(\frac{\left\|z_{q}-z_{p}\right\|}{\bar{d}}\right)>h^{\prime}(0)=\frac{2}{\pi} .
$$

All together we finally get

$$
\frac{2 \bar{d}}{\pi}<\bar{d} h^{\prime}\left(\frac{\left\|z_{q}-z_{p}\right\|}{\bar{d}}\right)=2\left\|z_{p}^{\perp}\right\|=2\left\|z_{p}\right\|<2\left\|z_{q}\right\|<2 r \leq \frac{2 d}{\pi} .
$$

Recalling that $d<\bar{d}$ this gives a contradiction and concludes the proof.

We go back now to the proof of Proposition 4.1. We have $\operatorname{diam} A_{1}=\operatorname{diam} \partial A_{1}$ (recall Remark 2.2). If we set

$$
\begin{aligned}
& C^{ \pm}=\left\{[z, t] \in \mathbb{H}^{n}:\|z\|=1 / \pi, 2 \pi t= \pm 1\right\}, \\
& D^{ \pm}=\left\{[z, t] \in \mathbb{H}^{n}:\|z\| \leq 1 / \pi, 2 \pi t= \pm 1\right\},
\end{aligned}
$$

we have $\partial A_{1} \subset A_{1}^{2} \cup D^{+} \cup D^{-} \subset A_{1}$ hence $\operatorname{diam} A_{1}=\operatorname{diam}\left(A_{1}^{2} \cup D^{+} \cup D^{-}\right)$. 
Let $p \in A_{1}^{2}$. We have $C^{+} \subset A_{1}^{2} \subset \bar{B}(0,1 / 2)$ hence $\operatorname{diam}\left(C^{+} \cup\{p\}\right) \leq 1$. If $\operatorname{diam}\left(D^{+} \cup\{p\}\right)>1$ it follows from Lemma 4.2 that $\left\|z_{p}\right\|<1 / \pi$ which contradicts the fact that $p \in A_{1}^{2}$. Hence $\operatorname{diam}\left(D^{+} \cup\{p\}\right) \leq 1$ for all $p \in A_{1}^{2}$. Since $\operatorname{diam} A_{1}^{2}=1$ it follows that $\operatorname{diam}\left(D^{+} \cup A_{1}^{2}\right)=1$. Similarly we have $\operatorname{diam}\left(D^{-} \cup A_{1}^{2}\right)=1$.

It thus only remains to check that $\operatorname{diam}\left(D^{-} \cup D^{+}\right) \leq 1$. Let $p \in D^{-}$. We have $C^{+} \cup\{p\} \subset A_{1}^{2} \cup D^{-}$hence $\operatorname{diam}\left(C^{+} \cup\{p\}\right) \leq 1$ by the previous argument. If $\operatorname{diam}\left(D^{+} \cup\{p\}\right)>1$ it follows from Lemma 4.2 that one can find $q \in D^{+}$ such that $z_{p}=z_{q}$ and $d(p, q)=\operatorname{diam}\left(D^{+} \cup\{p\}\right)>1$. On the other hand we have $-2 \pi t_{p}=2 \pi t_{q}=1$ and it follows from $(2.3)$ that $d(p, q)=1$ which gives a contradiction. Hence $\operatorname{diam}\left(D^{+} \cup\{p\}\right) \leq 1$ for any $p \in D^{-}$and since diam $D^{-} \leq 1$ we finally get $\operatorname{diam}\left(D^{-} \cup D^{+}\right) \leq 1$ as wanted.

The next lemma is an elementary remark that will be used later.

Lemma 4.3. Let $E \in \mathcal{R}$. Assume moreover that $E$ is symmetric with respect to the $\mathbb{C}^{n}$-plane, i.e., $[z,-t] \in E$ for all $[z, t] \in E$. Then

$$
E \subset\left\{[z, t] \in \mathbb{H}^{n}: 2\|z\| \leq \operatorname{diam} E, 2 \pi|t| \leq(\operatorname{diam} E)^{2}\right\} .
$$

Proof. Let $[z, t] \in E$. Since $E \in \mathcal{R}$ we have $[-z, t] \in E$ and it follows from (2.2) that $2\|z\|=d([-z, t],[z, t]) \leq \operatorname{diam} E$. We also have $[z,-t] \in E$ by assumption. It follows from $(2.3)$ that $(2 \pi|t|)^{1 / 2}=d([z, t],[z,-t]) \leq \operatorname{diam} E$ and this concludes the proof.

We give now the main result of this section.

Theorem 4.4. Let $E \in \mathcal{I}_{\mathcal{R}}$. Then $\mathrm{St} E \in \mathcal{I}_{\mathcal{R}}$ and $\operatorname{St} E=A_{\text {diam } E}$.

Proof. First we note that since $E$ is compact $\mathrm{St} E$ is also compact. Indeed, since $E$ is bounded, St $E$ is also obviously bounded. Next the fact that $E$ is compact implies that the map $z \in \pi(E) \mapsto \mathcal{L}^{1}(\{s \in \mathbb{R}:[z, s] \in E\})$ is upper semi-continuous. It follows that St $E$ is closed and hence compact. Since $E \in \mathcal{R}$, we have $\sigma(E)=E$, and it follows from Lemma 2.7 that $\operatorname{diam}(\operatorname{St} E) \leq \operatorname{diam} E$. On the other hand one has $\mathcal{L}^{2 n+1}(\mathrm{St} E)=\mathcal{L}^{2 n+1}(E)$. Since $E \in \mathcal{I}_{\mathcal{R}}$ and $\mathrm{St} E \in \mathcal{R}$, one actually has $\operatorname{diam}(\operatorname{St} E)=\operatorname{diam} E$ and St $E \in \mathcal{I}_{\mathcal{R}}$.

We set $\lambda=\operatorname{diam} E$ and we prove now that $\operatorname{St} E=A_{\lambda}$. Noting that $\pi(\operatorname{St} E)=$ $\pi(E)$ and taking into account the fact that $\mathrm{St} E \in \mathcal{I}_{\mathcal{R}}$ is also symmetric with respect to the $\mathbb{C}^{n}$-plane, it follows from Theorem 3.12 that

$$
\text { St } E=\left\{[z, t] \in \mathbb{H}^{n}: z \in \pi(E),|t| \leq f(z)\right\}
$$

for some continuous map $f: \pi(E) \rightarrow[0,+\infty)$ and that the set

$$
U=\{z \in \pi(E): f(z)>0\}
$$

is open in $\mathbb{C}^{n}$.

We have $\pi(E) \subset\left\{z \in \mathbb{C}^{n}: 2\|z\| \leq \lambda\right\}$ by Lemma 4.3 and we prove now that $f(z) \leq l_{\lambda}(\|z\|)$ for all $z \in \pi(E)$. In the case $\pi(E) \cap\left\{z \in \mathbb{C}^{n}: 2\|z\|=\lambda\right\} \neq \emptyset$, we note that since $U$ is open we must have $f(z)=0=l_{\lambda}(\|z\|)$ for any $z \in \pi(E)$ such 
that $2\|z\|=\lambda$. Next we know from Lemma 4.3 that $f(z) \leq \lambda^{2} /(2 \pi)=l_{\lambda}(\|z\|)$ for all $z \in \pi(E)$ such that $\|z\| \leq \lambda / \pi$.

It thus only remains to prove that $f(z) \leq l_{\lambda}(\|z\|)$ for $z \in \pi(E)$ such that $\lambda / \pi<\|z\|<\lambda / 2$. Given such a $z$ we assume, aiming to get a contradiction, that $f(z)>l_{\lambda}(\|z\|) \geq 0$ and set $p=\left[z, l_{\lambda}(\|z\|)\right] \in A_{\lambda} \cap \partial B(0, \lambda / 2)$. Since $f$ is continuous and $z$ belongs to the open set $U$, one can find an open set $V \subset$ $U \subset \pi(E)$ containing $z$ and such that $f(\|w\|)>l_{\lambda}(\|z\|)$ for all $w \in V$. Since $\left\{[w, s] \in \mathbb{H}^{n}: w \in V,|s| \leq f(\|w\|)\right\} \subset \operatorname{St} E$ it follows that $p \in \operatorname{int}($ St $E)$. Next, since $p \in \partial B(0, \lambda / 2)$ with $\|z\| \in(\lambda / \pi, \lambda / 2)$, we can write $p$ as

$$
p=\left[\chi e^{-i \varphi} \frac{\sin \varphi}{\varphi}, \frac{2 \varphi-\sin (2 \varphi)}{2 \varphi^{2}}\|\chi\|^{2}\right]
$$

for some $\chi \in \mathbb{C}^{n}$ such that $\|\chi\|=\lambda / 2$ and some $\varphi \in(-\pi / 2, \pi / 2)$ (see e.g. [10]). It follows from Lemma 1.11 in $[10]$ that $d(q, p)=2 d(0, p)=\lambda$ where

$$
q=\left[-\chi e^{i \varphi} \frac{\sin \varphi}{\varphi},-\frac{2 \varphi-\sin (2 \varphi)}{2 \varphi^{2}}\|\chi\|^{2}\right]
$$

Since $p \in \operatorname{int}($ St $E)$ and since the distance function from $q$ is an open map (see Lemma 2.1) we can find $p^{\prime} \in \mathrm{St} E$ such that $d\left(q, p^{\prime}\right)>\lambda$. On the other hand we have

$$
q=\left[e^{i(\pi+2 \varphi)} z,-l_{\lambda}(\|z\|)\right] .
$$

Since St $E \in \mathcal{R}$ is symmetric with respect to the $\mathbb{C}^{n}$-plane, we get that $q \in \operatorname{St} E$, i.e. a contradiction.

It follows that St $E \subset A_{\lambda}$. Since diam St $E=\operatorname{diam} E=\lambda=\operatorname{diam} A_{\lambda}$ by Proposition 4.1, and since $A_{\lambda} \in \mathcal{R}$ and St $E \in \mathcal{I}_{\mathcal{R}}$, we get that $\mathcal{L}^{2 n+1}\left(A_{\lambda} \backslash\right.$ St $\left.E\right)=0$. Being St $E$ closed, we obtain that $\operatorname{int}\left(A_{\lambda}\right) \backslash \operatorname{St} E=\operatorname{int}\left(A_{\lambda} \backslash\right.$ St $\left.E\right)=\emptyset$. It then follows that $A_{\lambda}=\overline{\operatorname{int}\left(A_{\lambda}\right)} \subset \operatorname{St} E$ and finally $\operatorname{St} E=A_{\lambda}$ as wanted.

We now show that $A_{\lambda}$ can be perturbed near the $t$-axis in such a way that the resulting set has the same volume and diameter as $A_{\lambda}$. As we shall see, the class of such perturbations is quite rich. It contains in particular rotationally and not rotationally invariant sets.

Proposition 4.5. There exists $r \in(0,1 / \pi)$ such that for every $\lambda>0$ and for any Lipschitz function $f: \mathbb{C}^{n} \rightarrow \mathbb{R}$ with compact support in $\left\{z \in \mathbb{C}^{n} ;\|z\|<\lambda r\right\}$ and with Lipschitz constant $\operatorname{Lip}(f)<\pi \lambda r / 4$, the set

$$
A_{\lambda, f}=\left\{[z, t] \in \mathbb{H}^{n} ;\|z\| \leq \lambda / \pi, 2 \pi|t-f(z)| \leq \lambda^{2}\right\} \cup A_{\lambda}^{2}
$$

satisfies $\mathcal{L}^{2 n+1}\left(A_{\lambda, f}\right)=\mathcal{L}^{2 n+1}\left(A_{\lambda}\right)$ and $\operatorname{diam} A_{\lambda, f}=\operatorname{diam} A_{\lambda}$.

Proof. The fact that $\mathcal{L}^{2 n+1}\left(A_{\lambda, f}\right)=\mathcal{L}^{2 n+1}\left(A_{\lambda}\right)$ is a consequence of the definition of $A_{\lambda, f}$ and of Fubini's theorem. To prove the last part of the lemma, we assume that $\lambda=1$ without loss of generality. Since $A_{1}^{2} \subset A_{1, f}$ and $\operatorname{diam} A_{1}^{2}=1$ we have $\operatorname{diam} A_{1, f} \geq 1=\operatorname{diam} A_{1}$. 
To complete the proof, we will show that the inequality $\operatorname{diam} A_{1, f} \leq 1=$ $\operatorname{diam} A_{1}$ holds up to a suitable choice of $r$. First, for a given $p \in \mathbb{H}^{n}$, we define

$$
h_{p}^{ \pm}(z)= \pm h\left(\left\|z-z_{p}\right\|\right)+t_{p}+2 \operatorname{Im} z_{p} \bar{z}
$$

so that $\bar{B}(p, 1)=\left\{[z, s] \in \mathbb{H}^{n}:\left\|z-z_{p}\right\| \leq 1, h_{p}^{-}(z) \leq s \leq h_{p}^{+}(z)\right\}$. We set $\kappa=h^{\prime}(0) / 2=1 / \pi$. one has

Claim 1. There exists $\bar{r} \in(0,1 / 2)$ such that, for all $p \in \mathbb{H}^{n}$ such that $\left\|z_{p}\right\| \leq \kappa / 4$,

$$
h_{p}^{+}(z) \geq h_{p}^{+}\left(z_{p}\right)+\kappa\left\|z-z_{p}\right\| \text { for all } z \in \mathbb{C}^{n} \text { such that }\left\|z-z_{p}\right\| \leq 2 \bar{r}
$$

and, similarly,

$$
h_{p}^{-}(z) \leq h_{p}^{-}\left(z_{p}\right)-\kappa\left\|z-z_{p}\right\| \text { for all } z \in \mathbb{C}^{n} \text { such that }\left\|z-z_{p}\right\| \leq 2 \bar{r} .
$$

Indeed one can find $\bar{r} \in(0,1 / 2)$ such that

$$
\left|h(r)-h(0)-h^{\prime}(0) r\right| \leq \frac{\kappa}{2} r
$$

for all $r \leq 2 \bar{r}$. Then we get

$$
\begin{aligned}
& h_{p}^{+}(z)=h\left(\left\|z-z_{p}\right\|\right)+t_{p}-2\left\langle i z_{p}, z-z_{p}\right\rangle \\
& \quad \geq h_{p}^{+}\left(z_{p}\right)+2 \kappa\left\|z-z_{p}\right\|-\frac{\kappa}{2}\left\|z-z_{p}\right\|-2\left\|z_{p}\right\|\left\|z-z_{p}\right\| \geq h_{p}^{+}\left(z_{p}\right)+\kappa\left\|z-z_{p}\right\|
\end{aligned}
$$

for all $p \in \mathbb{H}^{n}$ such that $\left\|z_{p}\right\| \leq \kappa / 4$ and all $z \in \mathbb{C}^{n}$ such that $\left\|z-z_{p}\right\| \leq 2 \bar{r}$. Here the scalar product is that of $\mathbb{R}^{2 n}$ after identifying points in $\mathbb{C}^{n}$ with points in $\mathbb{R}^{2 n}$. This gives (4.2). The proof of (4.3) is similar.

We set $p_{0}=[0,1 /(2 \pi)]$.

Claim 2. For all $\bar{r}>0$, there exists $\hat{r}>0$ such that

$$
\text { if } p \in B\left(p_{0}, \hat{r}\right) \cup B\left(p_{0}^{-1}, \hat{r}\right) \text { and } q \in \partial A_{1} \backslash B(p, 1) \text { then }\left\|z_{q}\right\|<\bar{r} \text {. }
$$

To prove this claim, we set

$$
K=\partial A_{1} \cap\left\{[z, t] \in \mathbb{H}^{n} ;\|z\| \geq \bar{r}\right\} \subset \bar{B}\left(p_{0}, 1\right) \cap \bar{B}\left(p_{0}^{-1}, 1\right)
$$

(recall that $p_{0}, p_{0}^{-1} \in A_{1}$ and $\operatorname{diam} A_{1}=1$ ). Since

$$
\partial B\left(p_{0}, 1\right) \cap \partial A_{1}=\left\{p_{0}^{-1}\right\} \quad \text { and } \quad \partial B\left(p_{0}^{-1}, 1\right) \cap \partial A_{1}=\left\{p_{0}\right\},
$$

we actually have

$$
K \subset B\left(p_{0}, 1\right) \cap B\left(p_{0}^{-1}, 1\right) .
$$

By compactness of $K$ and continuity of $p \mapsto \max _{q \in K} d(p, q)$, one can then find $\hat{r}>0$ such that $K \subset B(p, 1) \cap B\left(p^{\prime}, 1\right)$ for any $p \in B\left(p_{0}, \hat{r}\right)$ and $p^{\prime} \in B\left(p_{0}^{-1}, \hat{r}\right)$. This proves Claim 2. 
Fix $\bar{r} \in(0,1 / \pi)$ such that (4.2) and (4.3) hold for all $p \in \mathbb{H}^{n}$ satisfying $\left\|z_{p}\right\| \leq$ $\kappa / 4$. Then choose $\hat{r}>0$ such that (4.4) holds. Set $r=\min (\bar{r}, 2 \hat{r} / \pi, \kappa / 4)$. Let $f: \mathbb{C}^{n} \rightarrow \mathbb{R}$ be a Lipschitz function with compact support in $\left\{z \in \mathbb{C}^{n} ;\|z\|<r\right\}$ and with Lipschitz constant $\operatorname{Lip}(f)<\pi r / 4$.

We have $\|f\|_{\infty} \leq \operatorname{Lip}(f) r<\pi r^{2} / 4$ hence

$$
\begin{aligned}
\partial A_{1, f} \backslash \partial A_{1} \subset & \left\{[z, t] \in \mathbb{H}^{n}:\|z\|<r, 4\left|t-\frac{1}{2 \pi}\right|<\pi r^{2}\right\} \\
& \cup\left\{[z, t] \in \mathbb{H}^{n}:\|z\|<r, 4\left|t+\frac{1}{2 \pi}\right|<\pi r^{2}\right\} \\
\subset & B\left(p_{0}, \pi r / 2\right) \cup B\left(p_{0}^{-1}, \pi r / 2\right) \subset B\left(p_{0}, \hat{r}\right) \cup B\left(p_{0}^{-1}, \hat{r}\right) .
\end{aligned}
$$

Now we take $p \in \partial A_{1, f} \backslash \partial A_{1}$ and $q \in \partial A_{1, f}$. Without loss of generality, we also assume that $p \in B\left(p_{0}, \pi r / 2\right)$, the other case being analogous. Then

$$
p=\left[z_{p}, \frac{1}{2 \pi}+f\left(z_{p}\right)\right] .
$$

If $\left\|z_{q}\right\| \geq \bar{r} \geq r$ then $q \in \partial A_{1}$ and by (4.4) we get $d(p, q)<1$. If $\left\|z_{q}\right\|<\bar{r}<1 / \pi$ then

$$
t_{q}= \pm \frac{1}{2 \pi}+f\left(z_{q}\right) \text {. }
$$

If $t_{q}=\frac{1}{2 \pi}+f\left(z_{q}\right)$, since $\left\|z_{q}\right\|<\bar{r}$ and $\left|f\left(z_{q}\right)\right|<\pi r^{2} / 4 \leq \pi \bar{r}^{2} / 4$, we have $d\left(p_{0}, q\right)<$ $\pi \bar{r} / 2$. Therefore

$$
d(p, q)<\frac{\pi}{2}(r+\bar{r})<1
$$

If $t_{q}=-\frac{1}{2 \pi}+f\left(z_{q}\right)$ we note that $\left\|z_{p}\right\| \leq \kappa / 4$ and $\left\|z_{p}-z_{q}\right\| \leq r+\bar{r} \leq 2 \bar{r}$. Then by (4.3) we get

$$
\begin{aligned}
h_{p}^{-}\left(z_{q}\right) & \leq h_{p}^{-}\left(z_{p}\right)-\kappa\left\|z_{p}-z_{q}\right\|=-\frac{1}{\pi}+t_{p}-\kappa\left\|z_{p}-z_{q}\right\| \\
& \leq-\frac{1}{2 \pi}+f\left(z_{p}\right)-\operatorname{Lip}(f)\left\|z_{p}-z_{q}\right\| \leq-\frac{1}{2 \pi}+f\left(z_{q}\right)=t_{q} .
\end{aligned}
$$

Similarly, using (4.2),

$$
h_{p}^{+}\left(z_{q}\right) \geq h_{p}^{+}\left(z_{p}\right)+\kappa\left\|z_{p}-z_{q}\right\| \geq \frac{3}{2 \pi}+f\left(z_{q}\right)>t_{q} .
$$

Hence we have

$$
h_{p}^{-}\left(z_{q}\right) \leq t_{q} \leq h_{p}^{+}\left(z_{q}\right)
$$

that is $q \in \bar{B}(p, 1)$.

It follows that $d(p, q) \leq 1$ for all $p \in \partial A_{1, f} \backslash \partial A_{1}$ and $q \in \partial A_{1, f}$. Recalling that $\operatorname{diam}\left(\partial A_{1}\right)=1$ and that $\operatorname{diam} A_{1, f}=\operatorname{diam}\left(\partial A_{1, f}\right)$ this concludes the proof.

We get from this proposition the following two consequences:

Corollary 4.6. There exists $E \in \mathcal{I}_{\mathcal{R}}$ such that $p \cdot E \neq A_{\text {diam } E}$ for all $p \in \mathbb{H}^{n}$. 
In other words, although there is uniqueness modulo Steiner symmetrization with respect to the $\mathbb{C}^{n}$-plane for sets in $\mathcal{I}_{\mathcal{R}}$ with a given diameter, we have essential non uniqueness of sets in $\mathcal{I}_{\mathcal{R}}$.

Proof. Consider a set $A_{\lambda, f}$ given by Proposition 4.5 for some $\lambda>0$ and where $f \not \equiv 0$ is moreover chosen in such a way that $A_{\lambda, f} \in \mathcal{R}$. By Theorem 4.4, we have $A_{\lambda} \in \mathcal{I}_{\mathcal{R}}$. On the other hand, by Proposition 4.5 , we have $\mathcal{L}^{2 n+1}\left(A_{\lambda, f}\right)=$ $\mathcal{L}^{2 n+1}\left(A_{\lambda}\right)$ and $\operatorname{diam} A_{\lambda, f}=\operatorname{diam} A_{\lambda}$. Therefore $A_{\lambda, f} \in \mathcal{I}_{\mathcal{R}}$.

Let us prove that $p \cdot A_{\lambda, f} \neq A_{\lambda}$ for all $p \in \mathbb{H}^{n}$. First we note that if $F \in \mathcal{R}$ and $F$ is bounded then $p \cdot F \in \mathcal{R}$ if and only if $z_{p}=0$. Next it is straightforward from the analytic description of $A_{\lambda}$ and $A_{\lambda, f}$ that $p \cdot A_{\lambda, f} \neq A_{\lambda}$ for any $p \in \mathbb{H}^{n}$ such that $z_{p}=0$. This concludes the proof.

Another consequence of Theorem 4.4 and Proposition 4.5 is the existence of non-rotationally invariant isodiametric sets, even modulo left translations.

Corollary 4.7. There exists $E \in \mathcal{I}$ such that $p \cdot E \notin \mathcal{R}$ for all $p \in \mathbb{H}^{n}$.

Proof. If $\mathcal{I} \cap \mathcal{R}=\emptyset$ then there is nothing to prove. If $\mathcal{I} \cap \mathcal{R} \neq \emptyset$ then $\mathcal{I}_{\mathcal{R}}=$ $\mathcal{I} \cap \mathcal{R} \subset \mathcal{I}$. In particular it then follows from Theorem 4.4 that $A_{\lambda} \in \mathcal{I}$ for any $\lambda>0$. Let $A_{\lambda, f}$ be given by Proposition 4.5 where $f$ is moreover chosen in such a way that $A_{\lambda, f} \notin \mathcal{R}$. By Proposition 4.5 , we have $\mathcal{L}^{2 n+1}\left(A_{\lambda, f}\right)=\mathcal{L}^{2 n+1}\left(A_{\lambda}\right)$ and $\operatorname{diam} A_{\lambda, f}=\operatorname{diam} A_{\lambda}$, and hence $A_{\lambda, f} \in \mathcal{I}$. Next we check that $p \cdot A_{\lambda, f} \notin \mathcal{R}$ for all $p \in \mathbb{H}^{n}$. Assume by contradiction that $p \cdot A_{\lambda, f} \in \mathcal{R}$ for some $p \in \mathbb{H}^{n}$. Then we get in particular that $\rho_{\theta}\left(\pi\left(p \cdot A_{\lambda, f}\right)\right)=\pi\left(p \cdot A_{\lambda, f}\right)$ for all $\theta \in \mathbb{R}^{n}$ where $\rho_{\theta}$ is the rotation in $\mathbb{C}^{n}$ defined by $\rho_{\theta}(z)=\left(e^{i \theta_{1}} z_{1}, \ldots, e^{i \theta_{n}} z_{n}\right)$. On the other hand we have $\pi\left(p \cdot A_{\lambda, f}\right)=z_{p}+\left\{z \in \mathbb{C}^{n}: 2\|z\| \leq \lambda\right\}$. All together this implies that $z_{p}=0$. Then we get that $A_{\lambda, f}=p^{-1} \cdot\left(p \cdot A_{\lambda, f}\right)$ is the vertical left translation by $p^{-1}=\left[0,-t_{p}\right]$ of $p \cdot A_{\lambda, f} \in \mathcal{R}$ and hence belongs to $\mathcal{R}$, a contradiction.

\section{References}

[1] Agrachev, A., Barilari, D. And Boscain, U.: On the Hausdorff volume in subRiemannian geometry. Calc. Var. Partial Differential Equations 43 (2012), no. 3-4, $355-388$.

[2] Ambrosio, L. And Rigot, S.: Optimal mass transportation in the Heisenberg group. J. Funct. Anal. 208 (2004), no. 2, 261-301.

[3] Beer, G. And Villar, L.: Borel measures and Hausdorff distance. Trans. Amer. Math. Soc. 307 (1988), no. 2, 763-772.

[4] Besicovitch, A. S.: On the fundamental geometrical properties of linearly measurable plane sets of points (II). Math. Ann. 115 (1938), no. 1, 296-329.

[5] Bieberbach, L.: Uber eine Extremaleigenschaft des Kreises. J.-ber. Deutsch. Math.- Verein. 24 (1915), 247-250.

[6] Burago, Yu. D. And Zalgaller, V.A.: Geometric inequalities. Grundlehren der Mathematischen Wissenschaften 285, Springer Series in Soviet Mathematics, Springer-Verlag, Berlin, 1988. 
[7] Capogna, L., Danielli, D., Pauls, S. D. and Tyson, J. T.: An introduction to the Heisenberg group and the sub-Riemannian isoperimetric problem. Progress in Mathematics 259, Birkhäuser Verlag, Basel, 2007.

[8] Federer, H.: Geometric measure theory. Die Grundlehren der mathematischen Wissen schaften 153, Springer-Verlag, New York, 1969.

[9] Gaveau, B.: Principe de moindre action, propagation de la chaleur et estimées sous elliptiques sur certains groupes nilpotents. Acta Math. 139 (1977), no. 1-2, 95-153.

[10] Juillet, N.: Geometric inequalities and generalized Ricci bounds in the Heisenberg group. Int. Math. Res. Not. 13 (2009), 2347-2373.

[11] Preiss, D. And Tiser, J.: On Besicovitch's 1/2-problem. J. London Math. Soc. (2) 45 (1992), no. 2, 279-287.

[12] Rigot, S.: Isodiametric inequality in Carnot groups. Ann. Acad. Sci. Fenn. Math. 36 (2011), no. 1, 245-260.

[13] Stein, E. M.: Harmonic analysis: real-variable methods, orthogonality, and oscillatory integrals. Princeton Mathematical Series 43, Monographs in Harmonic Analysis III, Princeton University Press, Princeton, NJ, 1993.

[14] Urysohn, P.: Mittlere Breite and Volumen der konvexen Körper im n-dimensionalen Raume. Matem. Sb. SSSR 31 (1924), 477-486.

Received April 11, 2010

Gian Paolo Leonardi: Università di Modena e Reggio Emilia, Dipartimento di Scienze Fisiche, Informatiche e Matematiche, via Campi 213/b, 41100, Modena, Italy. E-mail: gianpaolo.leonardi@unimore.it

SÉverine Rigot: Université de Nice Sophia Antipolis, Laboratoire J.-A. Dieudonné, CNRS-UMR 7351, Parc Valrose, 06108 Nice cedex 02, France.

E-mail: rigot@unice.fr

Davide Vittone: Università di Padova, Dipartimento di Matematica,via Trieste 63, 35121 Padova, Italy.

E-mail: vittone@math.unipd.it

The first and third authors have been supported by the E.C. grant "GALA", MIUR, the GNAMPA grant "Metodi geometrici per analisi in spazi non Euclidei: spazi metrici doubling, gruppi di Carnot e spazi di Wiener" (2009). The first author is supported by the University of Modena and Reggio Emilia. The third author is supported by the University of Padova and by the Fondazione CaRiPaRo grant "Nonlinear Partial Differential Equations: models, analysis, and control-theoretic problems".

The second author wishes to thank the Department of Pure and Applied Mathematics of the University of Modena and Reggio Emilia, where part of the work was done, and also the GNAMPA project for financial support. The first and third authors are pleased to thank the Laboratoire J.-A. Dieudonné, Université de Nice Sophia-Antipolis, for its hospitality during the completion of a first draft of the paper.

The second author is supported by the ANR project GCM, program Blanc, project number NT09-504490. 